\title{
Intrinsic Functional Connectivity Patterns Predict Consciousness Level and Recovery Outcome in Acquired Brain Injury
}

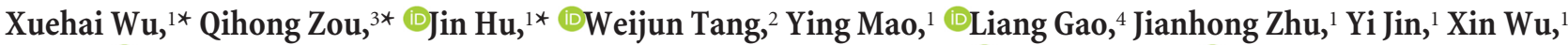 \\ Lu Lu, ${ }^{5}$ ○Yaojun Zhang, ${ }^{5}$ Yao Zhang, ${ }^{5}$ Zhengjia Dai, ${ }^{6,7}$ Jia-Hong Gao, ${ }^{3,8}{ }^{\circ}$ Xuchu Weng, ${ }^{\circ}$ Liangfu Zhou, ${ }^{1}$ \\ Georg Northoff, ${ }^{10}$ - Joseph T. Giacino, ${ }^{11}$ Yong He, ${ }^{6,7}$ and Yihong Yang ${ }^{12}$ \\ Departments of ${ }^{1}$ Neurosurgery and ${ }^{2}$ Radiology, Huashan Hospital, Fudan University, Shanghai 200040, People's Republic of China, ${ }^{3}$ Center for MRI \\ Research and Beijing City Key Laboratory of Medical Physics and Engineering, Peking University, Beijing 100871, People’s Republic of China, \\ ${ }^{4}$ Neurosurgical Department, Shanghai No.10 Hospital, Shanghai 200072, People's Republic of China, ${ }^{5}$ Neurosurgical Department, Huajia Hospital, Shanghai \\ 200433, People's Republic of China, ${ }^{6}$ State Key Laboratory of Cognitive Neuroscience and Learning and IDG/McGovern Institute for Brain Research, Beijing \\ Normal University, Beijing 100875, People's Republic of China, ${ }^{7}$ Center for Collaboration and Innovation in Brain and Learning Sciences, Beijing Normal \\ University, Beijing 100875, People's Republic of China, ${ }^{8} \mathrm{McGovern}$ Institute for Brain Research, Peking University, Beijing 100871, People's Republic of \\ China, ${ }^{9}$ Center for Cognition and Brain Disorders and The Affiliated Hospital, Hangzhou Normal University, Hangzhou, Zhejiang 310015, People's Republic \\ of China, ${ }^{10}$ Institute of Mental Health Research, University of Ottawa, Ottawa, Ontario K1Z 7K4, Canada, ${ }^{11}$ Spaulding Rehabilitation Hospital and \\ Department of Physical Medicine and Rehabilitation, Harvard Medical School, Boston, Massachusetts 02129, and ${ }^{2}$ Neuroimaging Research Branch, \\ National Institute on Drug Abuse, National Institutes of Health, Baltimore, Maryland 21224
}

For accurate diagnosis and prognostic prediction of acquired brain injury (ABI), it is crucial to understand the neurobiological mechanisms underlying loss of consciousness. However, there is no consensus on which regions and networks act as biomarkers for consciousness level and recovery outcome in ABI. Using resting-state fMRI, we assessed intrinsic functional connectivity strength (FCS) of whole-brain networks in a large sample of $99 \mathrm{ABI}$ patients with varying degrees of consciousness loss (including fully preserved consciousness state, minimally conscious state, unresponsive wakefulness syndrome/vegetative state, and coma) and 34 healthy control subjects. Consciousness level was evaluated using the Glasgow Coma Scale and Coma Recovery Scale-Revised on the day of fMRI scanning; recovery outcome was assessed using the Glasgow Outcome Scale 3 months after the fMRI scanning. One-way ANOVA of FCS, Spearman correlation analyses between FCS and the consciousness level and recovery outcome, and FCS-based multivariate pattern analysis were performed. We found decreased FCS with loss of consciousness primarily distributed in the posterior cingulate cortex/ precuneus (PCC/PCU), medial prefrontal cortex, and lateral parietal cortex. The FCS values of these regions were significantly correlated with consciousness level and recovery outcome. Multivariate support vector machine discrimination analysis revealed that the FCS patterns predicted whether patients with unresponsive wakefulness syndrome/vegetative state and coma would regain consciousness with an accuracy of $81.25 \%$, and the most discriminative region was the PCC/PCU. These findings suggest that intrinsic functional connectivity patterns of the human posteromedial cortex could serve as a potential indicator for consciousness level and recovery outcome in individuals with ABI.

Key words: acquired brain injury; hub; posterior cingulate cortex/precuneus; prediction; recovery outcome; resting state fMRI

\section{Significance Statement}

Varying degrees of consciousness loss and recovery are commonly observed in acquired brain injury patients, yet the underlying neurobiological mechanisms remain elusive. Using a large sample of patients with varying degrees of consciousness loss, we demonstrate that intrinsic functional connectivity strength in many brain regions, especially in the posterior cingulate cortex and precuneus, significantly correlated with consciousness level and recovery outcome. We further demonstrate that the functional connectivity pattern of these regions can predict patients with unresponsive wakefulness syndrome/vegetative state and coma would regain consciousness with an accuracy of $81.25 \%$. Our study thus provides potentially important biomarkers of acquired brain injury in clinical diagnosis, prediction of recovery outcome, and decision making for treatment strategies for patients with severe loss of consciousness. 


\section{Introduction}

Understanding the neurobiological mechanisms underlying loss of consciousness is essential for the diagnosis, treatment, and prognosis of acquired brain injury. Based on preclinical experiments and clinicopathological observations, the thalamocortical system has been considered crucial to consciousness (Zeman, 2001; Laureys et al., 2004; Långsjö et al., 2012; Schiff et al., 2014). However, there is no consensus on the key brain regions and corresponding networks that are essential to consciousness. Thus, the first goal of the current study was to investigate the neural mechanisms underlying consciousness by comparing a large cohort of acquired brain injury patients with varied levels of consciousness [fully preserved consciousness state (PC), minimally conscious state (MCS), unresponsive wakefulness syndrome (UWS)/vegetative state (VS), and coma] to healthy control subjects (CONs). Furthermore, it is important to accurately evaluate the consciousness status of acquired brain injury patients at early stages and to predict whether these patients will regain consciousness. Knowledge of the prognosis of acquired brain injury in its early phases would facilitate the optimization of treatment strategies and decision making with regard to withdrawing life-supporting measures if an unfavorable outcome is anticipated. Therefore, our second goal was to predict recovery outcome and promote early prognoses of favorable outcome.

Resting-state fMRI has emerged as a powerful tool for mapping intrinsic functional connectivity of the human brain, especially in clinical populations with low cooperation with task performance, such as patients with loss of consciousness (Sharp et al., 2014). Resting-state fMRI studies have demonstrated that hubs in the brain, which have high functional connectivity strength (FCS) and high rates of cerebral blood flow and glucose metabolism (Liang et al., 2013; Tomasi et al., 2013), serve to integrate diverse information sources (Achard et al., 2006; Buckner et al., 2009; Bullmore and Sporns, 2009; He and Evans, 2010; van den Heuvel and Sporns, 2013). These brain hubs are predominantly located in regions of the default mode network (DMN), including the posterior cingulate cortex (PCC)/precuneus (PCU), the medial prefrontal cortex, and the lateral temporal and parietal cortices (Raichle et al., 2001).

A theoretical framework based on information theory suggests that consciousness corresponds to the ability of the brain to integrate information (Tononi, 2004; Tononi and Koch, 2008). Several small-sample, resting-state fMRI studies using seedbased functional connectivity and independent component anal-

Received Jan. 27, 2015; revised July 5, 2015; accepted July 30, 2015.

Author contributions: Xuehai Wu, Q.Z., J.H., and L.Z. designed research; Xuehai Wu, J.H., W.T., Y.M., L.G., J.Z.,Y.J., Xin Wu, L.L., Yaojun Zhang, Yao Zhang, and Z.D. performed research; Q.Z., J.-H.G., X. Weng, G.N., J.T.G., Y.H., and Y.Y. contributed unpublished reagents/analytic tools; Q.Z. analyzed data; Xuehai Wu, Q.Z., G.N., Y.H., and Y.Y. wrote the paper.

This work was supported by the National Strategic Basic Research Program ("973") of China Grants 2015CB856400, 2012CB720700, 2010CB945500, 2012CB966300, and 2009CB941100; Natural Science Foundation of China Grants 81201142, 81225012, and 91432115; National Science and Technology 863 Program 2015AA020501; Shanghai Natural Science Foundation Grants 08411952000 and 10ZR1405400; the Canadian Institutes of Health Research (CIHR), EJLB Foundation-CIHR, the Michael Smith Foundation, and the Human Development Research Foundation. Y.Y. was supported by the Intramural Research Program of the National Institute on Drug Abuse, National Institutes of Health. We thank Drs. Gang Chen and Thomas Ross for providing help with statistical analyses; Drs.Yu-Feng Zang, Xi-Nian Zuo, and Zhifeng Kou for their insightful discussion; and Ms. Julia K. Brynildsen for linguistic editing of the manuscript.

The authors declare no competing financial interests.

* Xuehai Wu, Q.Z., and J.H. contributed equally to this work.

Correspondence should be addressed to either of the following: Liangfu Zhou, Neurosurgical Department, Huashan Hospital, Fudan University, Shanghai 200040, People's Republic of China, E-mail: Ifzhouc@126.com; or Yihong Yang, 251 Bayview Boulevard, Suite 200, Baltimore, MD 21224, E-mail: yihongyang@mail.nih.gov.

DOI:10.1523/JNEUROSCI.0415-15.2015

Copyright $\odot 2015$ the authors $\quad 0270-6474 / 15 / 3512933-15 \$ 15.00 / 0$ ysis have consistently shown that patients with acquired brain injury experience disrupted functional connectivity in the DMN, mainly in the PCC/PCU (Boly et al., 2009; Vanhaudenhuyse et al., 2010; Norton et al., 2012), a major hub node of the brain. Recently, another small-sample, resting-state fMRI study based on graph theory showed functional reorganization of hub regions in coma patients; cortical regions (e.g., PCU) that were major hubs of healthy brain networks had typically become non-hubs in comatose brain networks (Achard et al., 2012). On the basis of these studies, we hypothesized that FCS in brain hubs, such as the PCC/PCU, would be disrupted with varying degrees of consciousness loss, and that this disruption would serve as a potential biomarker for consciousness level and a predictor of recovery outcome in acquired brain injury.

\section{Materials and Methods}

Participants. The acquired brain injury patients were all recruited from the Neurosurgical Department, Huashan Hospital, Fudan University, and related rehabilitation hospitals of Huashan Hospital from February 2010 to August 2013. One hundred sixty-seven right-handed participants, including 133 patients with acquired brain injury and 34 healthy control subjects, participated in this study. Participants were excluded if they had any of the following clinical conditions: (1) a history of psychiatric or neurological illness before experiencing disorders of consciousness; (2) a history of medically documented brain injury; (3) a history of psychoactive drug consumption; and (4) current or previous drug or alcohol abuse. Thirty-four acquired brain injury patients were excluded due to excessive head motion during the functional scanning or excessive deformation in brain structure, leading to failure in spatial normalization (8 PC, 10 MCS, 11 UWS/VS and 5 COMA). The remaining 99 acquired brain injury patients and 34 control subjects were included in further analyses. Please refer to the Data preprocessing section for details of the patient exclusion procedure.

Patients were categorized into the following four subgroups (Schnakers, 2012): (1) PC group (40 patients; 24 males; mean age, $40.4 \pm$ 13.6 years), comprising patients who were able to communicate and had experienced brain injury, which was confirmed by cranial MRI and computer tomography scan; (2) MCS group (27 patients; 22 males; mean age, $42.5 \pm 14.0$ years), characterized by arousal/spontaneous eye-opening, fluctuating but reproducible behavioral signs of awareness, response to verbal orders, environmentally contingent smiling or crying, object localization and manipulation, sustained visual fixation and pursuit, verbalizations, intentional but unreliable communication, and emergence from MCS (ie, functional communication and functional object use; Giacino et al., 2002); (3) UWS/VS group (18 patients; 13 males; mean age, $42.0 \pm 15.2$ years), characterized by no evidence of awareness of self or environment and an inability to interact with others; no evidence of sustained, reproducible, purposeful, or voluntary behavioral responses to visual, auditory, tactile, or noxious stimuli; no evidence of language comprehension or expression; intermittent wakefulness manifested by the presence of sleep-wake cycles; sufficiently preserved hypothalamic and brain stem autonomic functions to permit survival with medical and nursing care; bowel and bladder incontinence; and variably preserved cranial nerve reflexes (pupillary, oculocephalic, corneal, vestibulo-ocular, and gag) and spinal reflexes (The Multi-Society Task Force on PVS, 1994); and (4) the coma group (14 patients; 10 males; mean age, $45.1 \pm 13.8$ years), characterized by no arousal/eye-opening, no behavioral signs of awareness, impaired spontaneous breathing, impaired brainstem reflexes, and no vocalizations of $>1 \mathrm{~h}$ (Plum and Posner, 1966).

The patients were scanned under a stable condition 4-667 d (84.9 \pm $100.9 \mathrm{~d}$ ) after the onset of acquired brain injury. The consciousness levels of the patients were assessed using the Glasgow Coma Scale (GCS; Teasdale and Jennett, 1974) and the Coma Recovery Scale-Revised (CRS-R; Giacino et al., 2004) on the day of the fMRI scanning. GCS includes three subscales addressing arousal level, motor function, and verbal abilities, which are added up and yield a total score ranging from 3 to 15 . The CRS-R includes six subscales addressing auditory, visual, motor, oromo- 
Table 1. Detailed demographic and clinical characteristics of the 99 patients

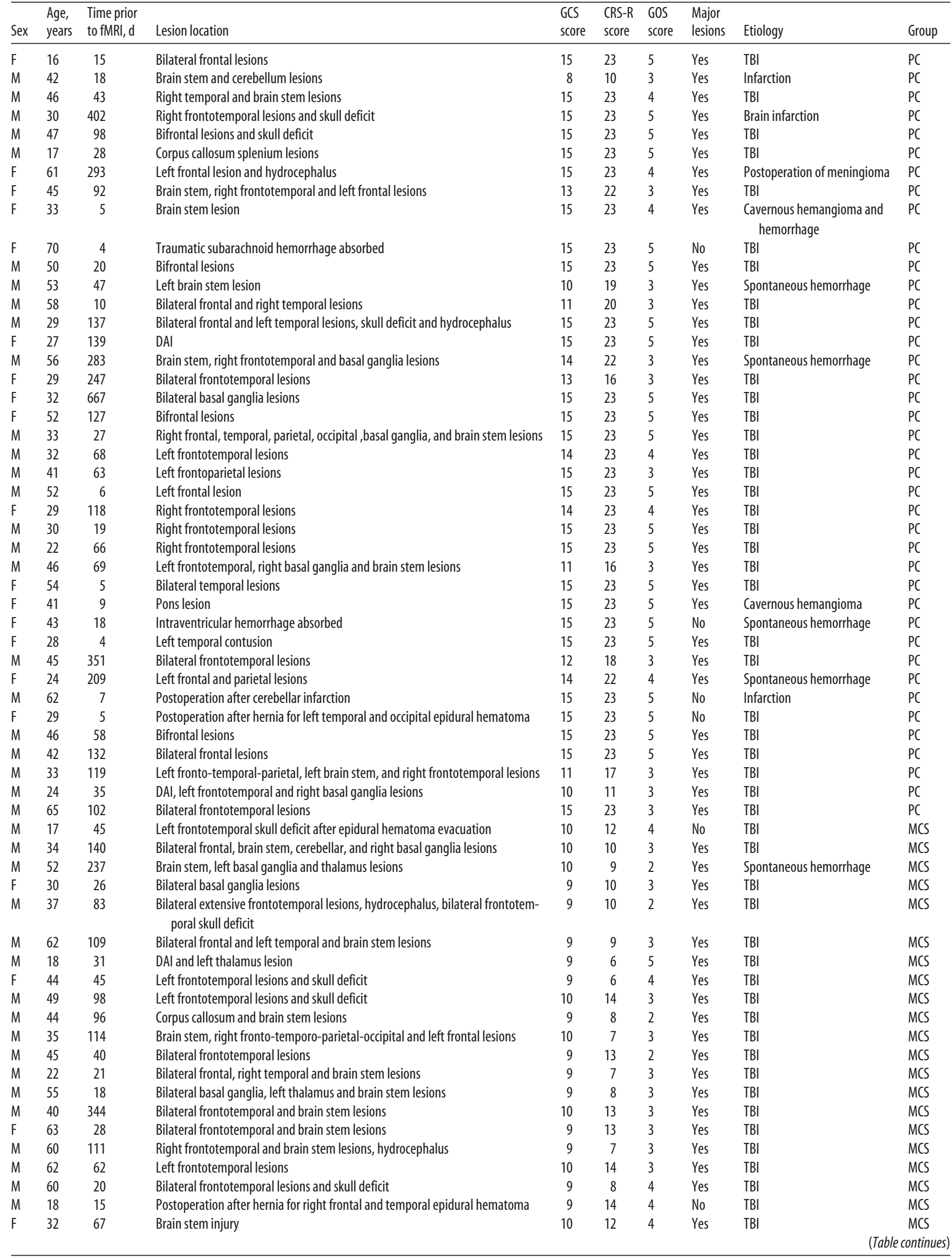


Table 1. Continued

\begin{tabular}{|c|c|c|c|c|c|c|c|c|c|}
\hline Sex & $\begin{array}{l}\text { Age, } \\
\text { years }\end{array}$ & $\begin{array}{l}\text { Time prior } \\
\text { to fMRI, d }\end{array}$ & Lesion location & $\begin{array}{l}\mathrm{GCS} \\
\text { score }\end{array}$ & $\begin{array}{l}\text { CRS-R } \\
\text { score }\end{array}$ & $\begin{array}{l}\text { GOS } \\
\text { score }\end{array}$ & $\begin{array}{l}\text { Major } \\
\text { lesions }\end{array}$ & Etiology & Group \\
\hline M & 48 & 53 & Bilateral frontotemporal and brain stem lesions, hydrocephalus & 9 & 7 & 3 & Yes & $\mathrm{TBI}$ & MCS \\
\hline M & 37 & 82 & Brain stem lesion & 9 & 13 & 3 & Yes & $\mathrm{TBI}$ & MCS \\
\hline M & 42 & 32 & Bilateral frontotemporal and bilateral basal ganglia lesions & 10 & 14 & 3 & Yes & $\mathrm{TBI}$ & MCS \\
\hline $\mathrm{F}$ & 44 & 79 & Bilateral frontal and temporal lesions & 8 & 7 & 2 & Yes & $\mathrm{TBI}$ & MCS \\
\hline M & 57 & 20 & Bilateral frontal and temporal lesions & 10 & 13 & 2 & Yes & $\mathrm{TBI}$ & MCS \\
\hline M & 40 & 148 & Right hemisphere and brain stem lesions & 8 & 6 & 3 & Yes & Spontaneous hemorrhage & MCS \\
\hline M & 25 & 74 & Left frontotemporal and brain stem lesions & 7 & 5 & 3 & Yes & $\mathrm{TBI}$ & UWS/VS \\
\hline M & 56 & 87 & $\begin{array}{l}\text { Left frontotemporal, occipital, brain stem, and right cerebellar lesions, left } \\
\text { frontotemporal skull deficit }\end{array}$ & 8 & 4 & 2 & Yes & $\mathrm{TBI}$ & UWS/VS \\
\hline M & 36 & 97 & Right basal ganglia, thalamus, right frontal, temporal and parietal lesions & 6 & 6 & 2 & Yes & $\mathrm{TBI}$ & UWS/VS \\
\hline M & 60 & 110 & $\begin{array}{l}\text { Diffused brain lesions, including brain stem, bilateral frontotemporal, basal } \\
\text { ganglia, thalamus, and right occipital lesions }\end{array}$ & 7 & 6 & 2 & Yes & TBI & UWS/VS \\
\hline M & 50 & 42 & DAl, right temporal lesion & 7 & 4 & 2 & Yes & $\mathrm{TBI}$ & UWS/VS \\
\hline $\mathrm{F}$ & 36 & 182 & DAI, right basal ganglia lesion & 9 & 5 & 2 & Yes & $\mathrm{TBI}$ & UWS/VS \\
\hline M & 59 & 44 & Bilateral frontotemporal, right occipital, and brain stem lesions & 8 & 4 & 2 & Yes & $\mathrm{TBI}$ & UWS/VS \\
\hline M & 11 & 216 & Left frontotemporal and brain stem lesions & 10 & 8 & 2 & Yes & $\mathrm{TBI}$ & UWS/VS \\
\hline M & 18 & 27 & Left temporal and right frontotemporal lesions, hydrocephalus & 8 & 5 & 4 & Yes & TBI & UWS/VS \\
\hline M & 42 & 21 & Left frontotemporal, right basal ganglia and brain stem lesions & 8 & 3 & 3 & Yes & $\mathrm{TBI}$ & UWS/VS \\
\hline $\mathrm{F}$ & 38 & 31 & Left frontotemporal, basal ganglia, right temporal, and brain stem lesions & 9 & 6 & 2 & Yes & $\mathrm{TBI}$ & UWS/VS \\
\hline $\mathrm{F}$ & 60 & 69 & Bilateral frontotemporal and brain stem lesions & 8 & 3 & 2 & Yes & TBI & UWS/VS \\
\hline M & 57 & 33 & Bilateral frontotemporal and brain stem lesions & 6 & 2 & 2 & Yes & $\mathrm{TBI}$ & UWS/VS \\
\hline $\mathrm{F}$ & 23 & 168 & $\mathrm{DAl}$ & 8 & 4 & 3 & No & $\mathrm{TBI}$ & UWS/VS \\
\hline M & 50 & 85 & Bilateral fronto-temporo-parietal and left occipital lesions & 9 & 4 & 2 & Yes & TBI & UWS/VS \\
\hline $\mathrm{F}$ & 52 & 52 & Brain stem and bilateral frontotemporal lesions & 7 & 4 & 2 & Yes & $\mathrm{TBI}$ & UWS/VS \\
\hline M & 36 & 39 & $\begin{array}{l}\text { Diffused bilateral frontotemporal, thalamus, midbrain and right occipital } \\
\text { lesions, bilateral frontotemporal skull deficit }\end{array}$ & 5 & 3 & 3 & Yes & $\mathrm{TBI}$ & UWS/VS \\
\hline M & 47 & 163 & Brain stem, right frontal and bilateral basal ganglia lesions & 8 & 5 & 3 & Yes & Spontaneous hemorrhage & UWS/VS \\
\hline M & 46 & 19 & $\begin{array}{l}\text { Left temporal, left basal ganglia, corpus callosum splenium and cingulum } \\
\text { lesions }\end{array}$ & 5 & 2 & 3 & Yes & $\mathrm{TBI}$ & Coma \\
\hline M & 63 & 8 & Left frontotemporal lesions & 3 & 0 & 1 & Yes & Spontaneous hemorrhage & Coma \\
\hline M & 56 & 20 & Brain stem lesion & 8 & 4 & 2 & Yes & TBI & Coma \\
\hline M & 58 & 180 & DAI, brain stem and bilateral frontotemporal lesions & 7 & 4 & 2 & Yes & $\mathrm{TBI}$ & Coma \\
\hline M & 37 & 21 & Brain stem, right frontotemporal and left frontal lesions & 7 & 3 & 3 & Yes & $\mathrm{TBI}$ & Coma \\
\hline M & 46 & 25 & DAl, brain stem, bilateral frontal and temporal lesions & 6 & 2 & 4 & Yes & $\mathrm{TBI}$ & Coma \\
\hline $\mathrm{F}$ & 38 & 65 & DAl, left temporal lesion & 8 & 6 & 3 & Yes & $\mathrm{TBI}$ & Coma \\
\hline $\mathrm{F}$ & 67 & 10 & Brain stem and left frontotemporal lesions & 6 & 3 & 2 & Yes & TBI & Coma \\
\hline M & 47 & 13 & Pons lesion & 7 & 4 & 3 & Yes & Pons demyelination & Coma \\
\hline M & 44 & 71 & Brain stem lesion & 6 & 2 & 2 & Yes & $\mathrm{TBI}$ & Coma \\
\hline $\mathrm{F}$ & 39 & 27 & Brain stem lesion & 8 & 6 & 2 & Yes & TBI & Coma \\
\hline $\mathrm{F}$ & 49 & 47 & Brain stem lesion & 8 & 5 & 3 & Yes & Spontaneous hemorrhage & Coma \\
\hline M & 23 & 17 & Left hemisphere, right frontal, and brain stem lesions & 8 & 6 & 4 & Yes & Spontaneous hemorrhage & Coma \\
\hline M & 18 & 18 & DAl, brain stem lesion & 6 & 3 & 3 & Yes & TBI & Coma \\
\hline
\end{tabular}

DAl, diffused axonal injury; TBI, traumatic brain injury; $F$, female; $M$, male.

Table 2. Demographic and clinical characteristics of the healthy control subjects and acquired brain injury patients

\begin{tabular}{|c|c|c|c|c|c|c|c|}
\hline & $\mathrm{CON}$ & $P C$ & MCS & UWS/VS & Coma & $p$ value (5 groups) & $p$ value (4 ABI groups) \\
\hline Gender & $22 \mathrm{M}, 12 \mathrm{~F}$ & $24 \mathrm{M}, 16 \mathrm{~F}$ & $22 \mathrm{M}, 5 \mathrm{~F}$ & $13 \mathrm{M}, 5 \mathrm{~F}$ & $10 \mathrm{M}, 4 \mathrm{~F}$ & 0.43 & 0.30 \\
\hline Age, years & $40.2 \pm 10.7$ & $40.4 \pm 13.6$ & $42.5 \pm 14.0$ & $42.0 \pm 15.2$ & $45.1 \pm 13.8$ & 0.77 & 0.74 \\
\hline Time prior to fMRI after injury, $d$ & & $104.1 \pm 136.3$ & $80.1 \pm 73.5$ & $85.6 \pm 59.7$ & $38.6 \pm 45.1$ & & 0.22 \\
\hline GCS score & & $14.0 \pm 1.8$ & $9.3 \pm 0.6$ & $7.7 \pm 1.2$ & $6.6 \pm 1.4$ & & $<0.001$ \\
\hline CRS-R score & & $21.5 \pm 3.2$ & $10.0 \pm 2.9$ & $4.5 \pm 1.4$ & $3.6 \pm 1.8$ & & $<0.001$ \\
\hline GOS score & & $4.3 \pm 0.9$ & $3.0 \pm 0.8$ & $2.4 \pm 0.6$ & $2.6 \pm 0.8$ & & $<0.001$ \\
\hline
\end{tabular}

Values are reported as the mean $\pm S D$, unless otherwise indicated. $A B l$, acquired brain injury; $F$, female; $M$, male. $p$ values $<0.05$ are labeled in bold.

tor, communication, and arousal functions, which are summed to yield a total score ranging from 0 to 23 . The recovery outcome was assessed using the Glasgow Outcome Scale (GOS; Jennett and Bond, 1975) at 3 months after fMRI scanning. The GOS provides a measurement of outcome ranging from 1 to 5 (1, dead; 2, vegetative state/severe disability; 3 , able to follow commands/unable to live independently/moderate disability; 4, able to live independently/unable to return to work or school; 5, good recovery/able to return to work or school). Thus, clinical indices were all measured at a single time point, though the GOS score was evaluated during a follow-up period that occurred 3 months after the imaging and the other clinical indices. This study was approved by the Medical Research Ethics Committee and the Institutional Review Board of the Huashan Hospital, and informed consent was obtained from each participant or from their legal representative. Detailed demographic and clinical characteristics of patients are listed in Table 1, while summarized information of the five groups of participants is provided in Table 2.

Data acquisition. All participants were scanned using a 3 tesla Siemens Verio scanner with a 12-channel head coil in Huashan Hospital, Fudan 
University. Resting-state fMRI images were collected using an echoplanar imaging sequence $(\mathrm{TR}=2000 \mathrm{~ms}$; TE $=35 \mathrm{~ms}$; flip angle $=90^{\circ}$; number of slices $=33$; slice thickness $=4 \mathrm{~mm}$; gap $=0 \mathrm{~mm}$; matrix $=$ $64 \times 64)$. The participants were scanned with the following two in-plane resolutions due to different field-of-view selections by different MRI scanner operators: (1) in-plane resolution $=4 \times 4 \mathrm{~mm}^{2}$ in $19 \mathrm{CONs}$, and $24 \mathrm{PC}, 18$ MCS, 14 UWS/VS, and 7 coma patients; and (2) in-plane resolution $=3.281 \times 3.281 \mathrm{~mm}^{2}$ in 15 CONs, and 16 PC, 9 MCS, 4 UWS/VS, and 7 coma patients. During the data acquisition, participants lay quietly in the scanner. The resting-state fMRI scanning lasted for $400 \mathrm{~s}$ and resulted in 200 volumes for each participant. The healthy control subjects and communicative acquired brain injury patients were instructed to keep their eyes closed during the resting-state fMRI scan. A set of T2-weighted images was acquired from each participant to assess focal brain injury using the following parameters: $\mathrm{TR}=6670 \mathrm{~ms}$; $\mathrm{TE}=95 \mathrm{~ms}$; flip angle $=90^{\circ}$; matrix $=256 \times 256$; number of axial slices $=33$; thickness $=4 \mathrm{~mm}$ and voxel size $=1 \times 1 \times 4 \mathrm{~mm}^{3}$. The T2 images were reviewed by a senior neuroradiologist.

Data preprocessing. Resting-state fMRI data were preprocessed (Power et al., 2014) using the Analysis of Functional NeuroImages software package (Cox, 1996), Statistical Parametric Mapping (Wellcome Department of Imaging Neuroscience, University College London, London, UK), and Matlab (MathWorks). The preprocessing steps for the restingstate fMRI data included removal of the first four volumes, slice timing and head motion correction, spatial normalization to a template at the Montreal Neurological Institute space, linear trend removal, nuisance signal regression, temporal bandpass filtering $(0.009-0.08 \mathrm{~Hz})$, and spatial smoothing with a $6 \mathrm{~mm}$ Gaussian kernel (Power et al., 2014). During nuisance signal regression, 30 regressors, including 24 head motionrelated regressors derived by Volterra expansion (Friston et al., 1996), 3 tissue-based average signals from CSF, white matter, and whole-brain and first derivatives of tissue-based signals, were regressed out.

Framewise displacement of head movement was evaluated with the sum of the absolute values of the six motion parameter derivatives, as follows:

$$
F D_{i}=\left|\Delta d_{i x}\right|+\left|\Delta d_{i y}\right|+\left|\Delta d_{i z}\right|+\left|\Delta \alpha_{i}\right|+\left|\Delta \beta_{i}\right|+\left|\Delta \gamma_{i}\right|,
$$

where $\Delta d_{i x}=d_{(i-1) x}-d_{i x}$. The rotational displacements were converted from radians to millimeters by calculating the displacement on the surface of a sphere of radius $50 \mathrm{~mm}$ (Power et al., 2012; Liang et al., 2013). To moderate the effects of head motion on estimates of functional connectivity, we censored volumes within each participant's restingstate fMRI time series that were associated with sudden head motion (i.e., the volumes with $F D_{i}>0.5 \mathrm{~mm}$ ). To minimize the influence of head motion, we further excluded subjects whose $F D=\frac{1}{N-1} \Sigma F D_{i}$ $>0.25 \mathrm{~mm}$ or total length of data were $<4.5 \mathrm{~min}$ (135 TRs) after "scrubbing."

Of the 133 acquired brain injury patients, 94 (70.7\%) had volumes that underwent scrubbing. Twenty-one acquired brain injury patients with average framewise displacement $>0.25 \mathrm{~mm}$ or volumes of data $<135$ after motion censoring were excluded from further analyses. Thus, 112 acquired brain injury patients were included in subsequent analyses. Though data from 16 of the 34 control subjects $(47.1 \%)$ were motion censored according to the above criteria, none of them were excluded. Data from 13 acquired brain injury patients failed to meet spatial normalization criteria and were excluded from further analyses. After data preprocessing, we had resting-state functional scans from 99 acquired brain injury patients and $34 \mathrm{CONs}$, and there was no significant differ- ence in average framewise displacement between the patient and control groups. Of note, after temporal scrubbing, the number of volumes left for FCS analysis varied across subjects and was dependent upon the motion curves for each subject. We observed a significant main effect of group on volume number across five groups $(p=0.005)$, whereby MCS and UWS/VS patients had fewer volumes than CONs. In the following section, we controlled the motion effect in our validation analyses and showed that when data for all participants were cut to the same length, our main findings were minimally changed.

Network construction. We constructed graphs at a voxel level, where nodes represented brain voxels and edges represented intervoxel functional connectivity. The time series for each voxel was extracted from the preprocessed resting-state fMRI data to calculate a correlation matrix $r=$ $\left(r_{i, j}\right) ; 1 \leq i \leq N ; 1 \leq j \leq N$ ( $N$ is the number of voxels), where $r_{i, j}$ is the temporal Pearson's correlation of time series between the $i$ th and $j$ th voxels and measures the similarity of resting-state fMRI signal between the two voxels. A threshold of correlation, $r_{0}$, was estimated with Bonferroni corrected $p=0.05$ [i.e., original $p=0.05 /(N *(N-1) / 2)$ ]. It was used to threshold the correlation matrix into an adjacency matrix $A=$ $\left(a_{i, j}\right) ; 1 \leq i \leq N ; 1 \leq j \leq N$, as follows:

$$
a_{i j}=\left\{\begin{array}{l}
0,\left|r_{i j}\right|<r_{0} \\
\left|r_{i j}\right|,\left|r_{i j}\right| \geq r_{0} .
\end{array}\right.
$$

To exclude artifactual correlations from non-gray matter voxels, we restricted our voxelwise connectivity strength analyses to a predefined gray matter mask with gray matter tissue probability of $>20 \%$. The gray matter tissue probability template was released as a part of tissue priors in SPM8 (http://www.fil.ion.ucl.ac.uk/spm/software/spm8).

Functional connectivity strength analysis. We computed the weighted FCS for each nodal voxel, which was defined as the sum of weights from edges connecting to a given node. According to the adjacency matrix of a graph, FCS can be computed by Equation 3. FCS has been widely used to examine node characteristics of resting-state networks (Buckner et al., 2009; Bullmore and Sporns, 2009; He et al., 2009; Cole et al., 2010; Wang et al., 2011; Zuo et al., 2012) and has a demonstrated close relationship with cerebral blood flow (Liang et al., 2013) and cerebral metabolic rate of glucose (Tomasi et al., 2013).

$$
\operatorname{FCS}(i)=\sum_{j=1}^{N} a_{i j} .
$$

Weighted FCS was adopted for our main analyses, as weighted networks were numerically more reliable in the face of noise in functional connec- 


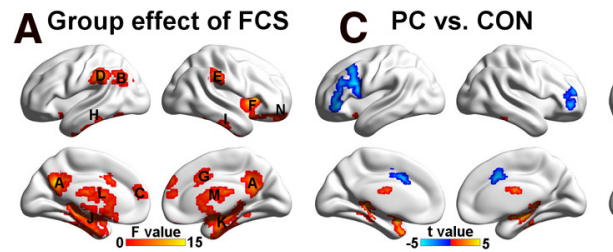

B Fingerprint plots of FCS

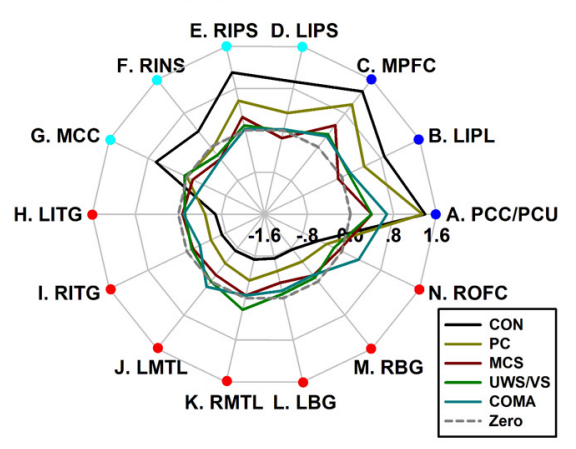

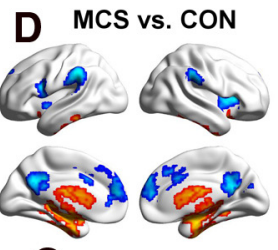

G MCs vs. PC
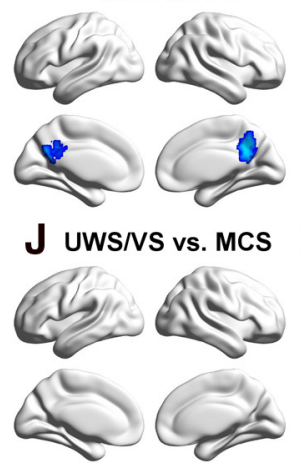

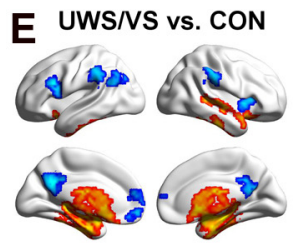

H uWs/vs vs. PC
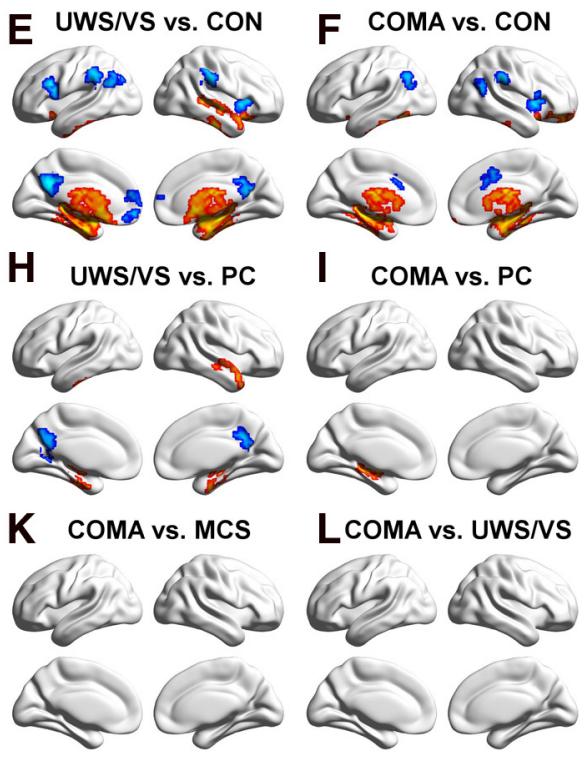

Figure 2. Group differences in FCS. A, Main group effect of FCS among the five groups ( $p<0.05$ corrected, with uncorrected $p<0.01$ for each voxel and a minimum of 84 voxels in each cluster). The largest region was composed of multiple brain areas and was decomposed into several subregions, including the right anterior insula, left inferior temporal gyrus, right inferior temporal gyrus, left medial temporal lobe, right medial temporal lobe, left basal ganglia, right basal ganglia, and right orbital frontal cortex. B, Fingerprint plots of the mean fitted FCS (FCS with covariates such as the age and gender removed) across the five groups for each of the regions that showed main group effects. Regions overlapping the DMN are labeled in blue, while those overlapped with the $S N / E C N$ were labeled in cyan. 0 ther regions that showed increased FCS with decreased consciousness were labeled in red. $C-L$, Post hoc comparisons of 10 pairs of groups ( $p<0.05$ corrected). Two-sample $t$-maps highlighting the difference between PC patients and CONs $(\boldsymbol{C})$, MCS patients and CONs $(\boldsymbol{D})$, UWS/VS patients and CONs $(\boldsymbol{E})$, coma patients and CONs $(\boldsymbol{F})$, MCS and PC patients $(\boldsymbol{G})$, UWS/VS and $P C$ patients $(\boldsymbol{H})$, coma and PC patients $(\boldsymbol{I})$, UWS/VS and MCS patients $(\boldsymbol{J})$, coma and MCS patients $(\boldsymbol{K})$, and coma and UWS/VS patients $(\boldsymbol{L})$ are shown. To note, there was no significant difference between UWS/VS and MCS patients $(\boldsymbol{J})$, between coma and MCS patients $(\boldsymbol{K})$, or between coma and UWS/VS patients (L). LIPL, Left inferior parietal lobule; MPFC, medial prefrontal cortex, LIPS, left intraparietal sulcus; RIPS, right intraparietal sulcus; RINS, right anterior insula; MCC, middle cingulate cortex; LITG, left inferior temporal gyrus; RITG, right inferior temporal gyrus; LMTL, left medial temporal lobe; RMTL, right medial temporal lobe; LBG, left basal ganglia; RBG, right basal ganglia; and ROFC, right orbital frontal cortex.

tivity (Wang et al., 2011), and FCS values were standardized to $z$-scores before statistical analysis (Liang et al., 2013).

Statistical analysis. To test main group differences, one-way ANOVA of the FCS was conducted for the five groups (CONs, and PC, MCS, UWS/VS, and coma patients). The effects of age, gender, resolution type, number of volumes remaining, and average framewise displacement after motion scrubbing were regressed out before ANOVA. Spearman's correlation coefficient was calculated to assess the relationships between FCS and the consciousness level, using both the GCS and CRS-R, in all the acquired brain injury patients. To test whether FCS could correlate with recovery outcome, Spearman's correlation analysis was performed on recovery outcome values indexed by the GOS. The effects of age, gender, resolution type, number of volumes remaining, average framewise displacement after motion scrubbing, and time elapsed before fMRI after injury were controlled for before the Spearman's correlation analysis. We used a standard approach implemented in AFNI (AlphaSim; 10,000 Monte Carlo simulations) to correct for multiple comparisons (Forman et al., 1995; Wang et al., 2012; Zou et al., 2013). By iterating the process of random image generation, spatial correlation of voxels, thresholding, and cluster identification, the AlphaSim program provides an estimate of the overall significance level achieved for various combinations of individual voxel probability threshold and cluster size threshold (Forman et al., 1995). Using this program, a corrected significance level of $p<0.05$ for the resultant statistical maps was obtained by clusters with a minimum number of 84 voxels at an uncorrected individual voxel height threshold of $p<0.05$. This multiple comparisons correction approach was consistently applied to all the statistical analyses, including the one-way ANOVA and Spearman's correlation analysis.

Multivariate support vector machine discrimination analysis. Three months after the MRI scanning, the GOS was rated for each patient. Patients with a GOS score of no less than 3 were defined as awakened, while those with a GOS score of $<3$ were defined as nonawakened. To further investigate whether patients in UWS/VS and coma states who had awakened (GOS score of $\geq 3$ ) within 3 months of the fMRI scanning could be differentiated from those who did not awaken (GOS score of
$<3$ ), we performed multivariate pattern analysis on FCS using a support vector machine approach. This analysis included the following two main steps: feature selection and multivariate classification. The generalization performance of the classifier was evaluated using a leave-one-out crossvalidation (LOOCV) scheme. Feature selection is an effective way to identify relevant features with the greatest discriminative power for classification, which improves the final classification performance (Dosenbach et al., 2010; Dai et al., 2012). In this study, features with significant differences ( $p<0.05$, uncorrected) both between the awakened and nonawakened patients and among the five groups in the training set (with a test UWS/VS or coma patient excluded in each iteration of the LOOCV) were selected. Notably, feature selection was always performed on the training data only, which avoided overfitting of the classifier. Thus, the features selected were slightly different from iteration to iteration of the LOOCV. A linear support vector machine was used for classification that allows direct extraction of the discriminating weight for each region (Ecker et al., 2010). The LIBSVM toolbox for MatLab was used to perform the classification (https://www.csie.ntu.edu.tw/ cjlin/ libsvm/). A discrimination map was generated for each LOOCV iteration, and a final discrimination map showing the most discriminative features was obtained by averaging the discrimination maps across all iterations. During each LOOCV iteration, we determined whether the test sample was correctly predicted based on the multivariate classifier using the training data. Accuracy, sensitivity, and specificity were calculated to quantify the performance of the classifier for all 32 iterations of the LOOCV. Sensitivity denotes the ratio of nonawakened patients correctly predicted, while specificity represents the proportion of awakened patients correctly predicted. The ratio of all correctly predicted patients was quantified for accuracy. Notably, the effects of age, gender, resolution type, number of volumes remaining, average framewise displacement after motion scrubbing, and time elapsed before fMRI after injury were controlled for before the multivariate pattern analysis. Furthermore, a more detailed analysis was performed using leave-two-out cross-validation (LTOCV). Of note, the LTOCV resulted in a smaller sample size $(\mathrm{N}-2$; i.e., 30 in the 
current study) than the LOOCV ( $\mathrm{N}-1$; i.e., 31 in the current study) in the training data for feature selection, which might have reduced the performance of multivariate pattern analysis.

Additionally, we performed univariate discriminative analysis by plotting the receiver operating characteristic curve of each demographic and clinical variable, including age, gender, GCS score, CRS-R score, and the time elapsed before fMRI after injury.

Seed-based resting-state functional connectivity analysis. The overlapped region showing a group main effect of FCS, correlation with consciousness level and recovery, and discriminative power was chosen as a "seed" to perform seed-based resting-state functional connectivity analysis. This analysis was adopted to identify specific effects (intranetwork effects, internetwork effects, or both) involved in the abnormal FCS found in the acquired brain injury patients (i.e., to determine which brain regions possess abnormal functional connectivity with the seed). We calculated the Pearson's correlation coefficient between the PCC/ PCU seed time series and every other voxel in the brain, and transformed the correlation coefficient values into $z$-values using Fisher's $r$-to- $z$ transformation. Using the PCC/PCU connectivity maps, the same statistical analyses as used for the FCS were performed, including (1) one-way ANOVA on five participant groups; (2) Spearman's correlation analyses with the GCS, CRS-R, and GOS; and (3) multivariate support vector machine analysis of the UWS/VS plus coma patients to characterize awakened or nonawakened status.

Validation analyses. To validate our FCS results, we adopted several procedures to assess the effect of different preprocessing and analytic strategies. (1) The effect of network type: besides the weighted network analysis, we also implemented a binary network analysis. For a binary graph, the FCS of a node is calculated as the number of edges connecting to the node. We calculated the binary FCS with edge significance thresholded at a Bonferroni corrected $p=0.05$. We then repeated the same analyses used for weighted FCS, including ANOVA and Spearman's correlation analyses. (2) The effect of data length; after temporal scrubbing of the fMRI data, the number of volumes left for FCS analysis varied across subjects, depending upon the motion curves of each subject ( $p=$ 0.005 for ANOVA across five groups, with MCS and UWS/VS patients having fewer volumes than CONs). We further tested whether data length [whole data after scrubbing with varied number of volumes vs minimum data with the same number of volumes (135 TRs)] would affect our findings. We adopted a strategy that volumes with the largest framewise displacement were removed and the number of volumes left was 135 for each subject (Belcher et al., 2013). We then calculated the weighted FCS based on the data scrubbed to the same length, and conducted statistical analyses, including ANOVA and Spearman's correlation analyses. (3) The effect of negative connections; the removal of the global mean signal causes negative connections, the physiological mechanism of which is still under debate (Fox et al., 2009; Murphy et al., 2009). We investigated

\section{A FCS correlated with GCS}
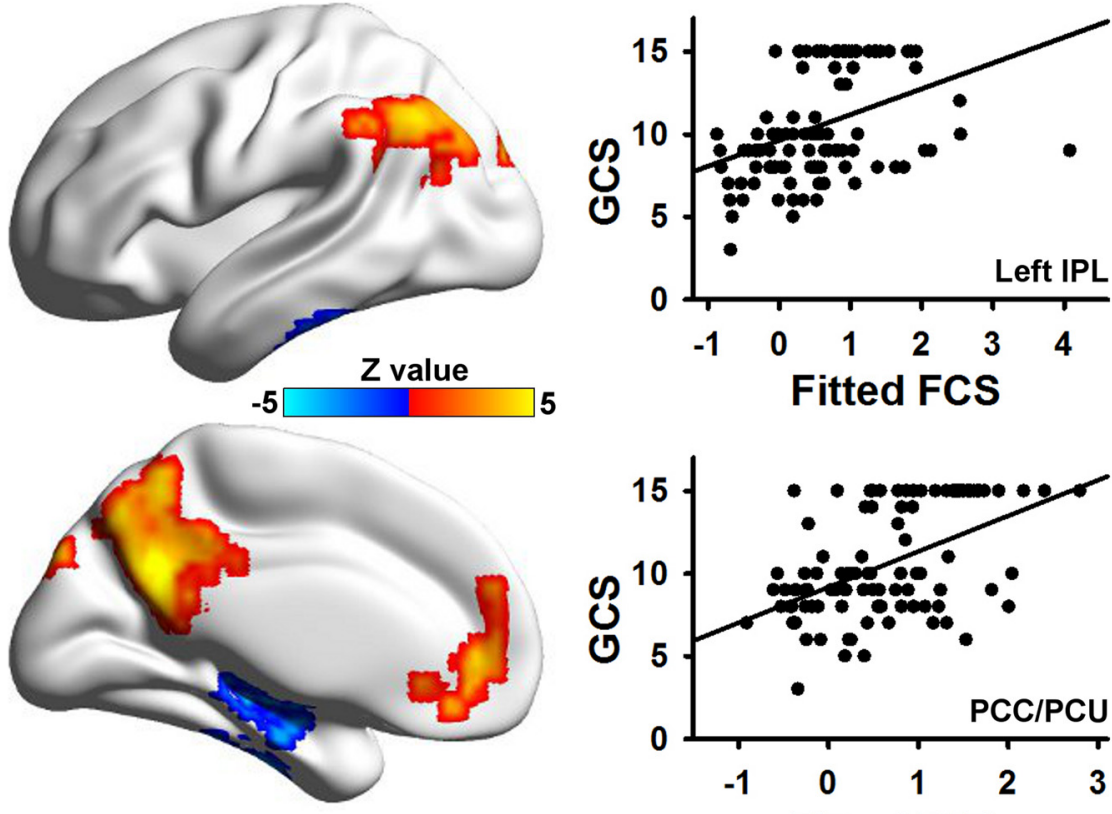

Fitted FCS

\section{B FCS correlated with CRS-R}
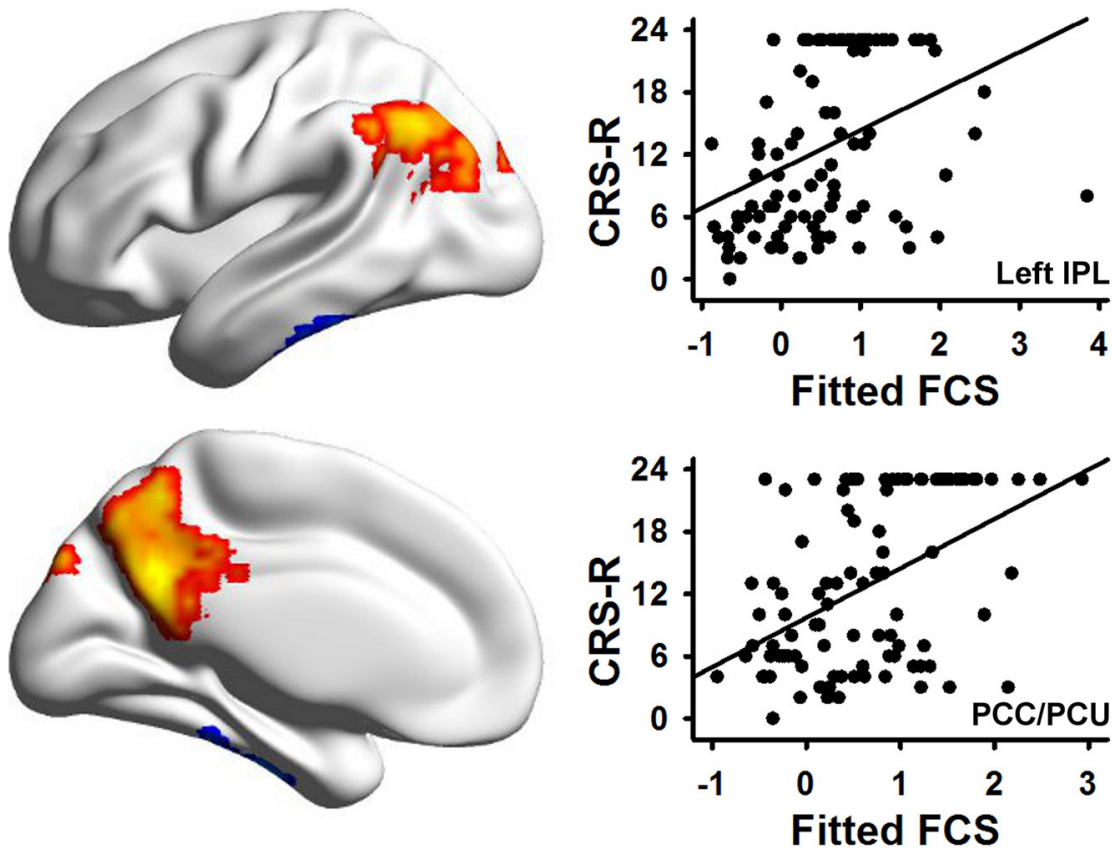

Figure 3. A, The relationship between FCS and the GCS score. Six regions showed a significant Spearman's correlation based on voxelwise analysis after correction $(p<0.05)$. Scatter plots between the mean fitted FCS (FCS with covariates such as age, gender, and time elapsed before fMRI after injury removed) in two representative regions (the PCC/PCU and the left IPL) and the GCS score are shown. $\boldsymbol{B}$, The relationship between FCS and the CRS-R score. Four regions showed significant correlation based on voxelwise analysis after correction $(p<0.05)$. Scatter plots between the mean fitted FCS in two representative regions (the PCC/PCU and the left IPL) and the (RS-R score are shown. LIPL, Left inferior parietal lobule. whether or not including negative correlations during FCS calculations would affect the findings. (4) The effect of global mean signal removal; as mentioned above, the removal of the global mean signal causes spurious anticorrelations. We additionally investigated whether or not using the global mean signal as a confound during FCS calculations would affect the findings. (5) The effect of local short-distance correlations; the FCS values calculated above would be influenced by both local short-distance 


\section{A FCS correlated with GOS}
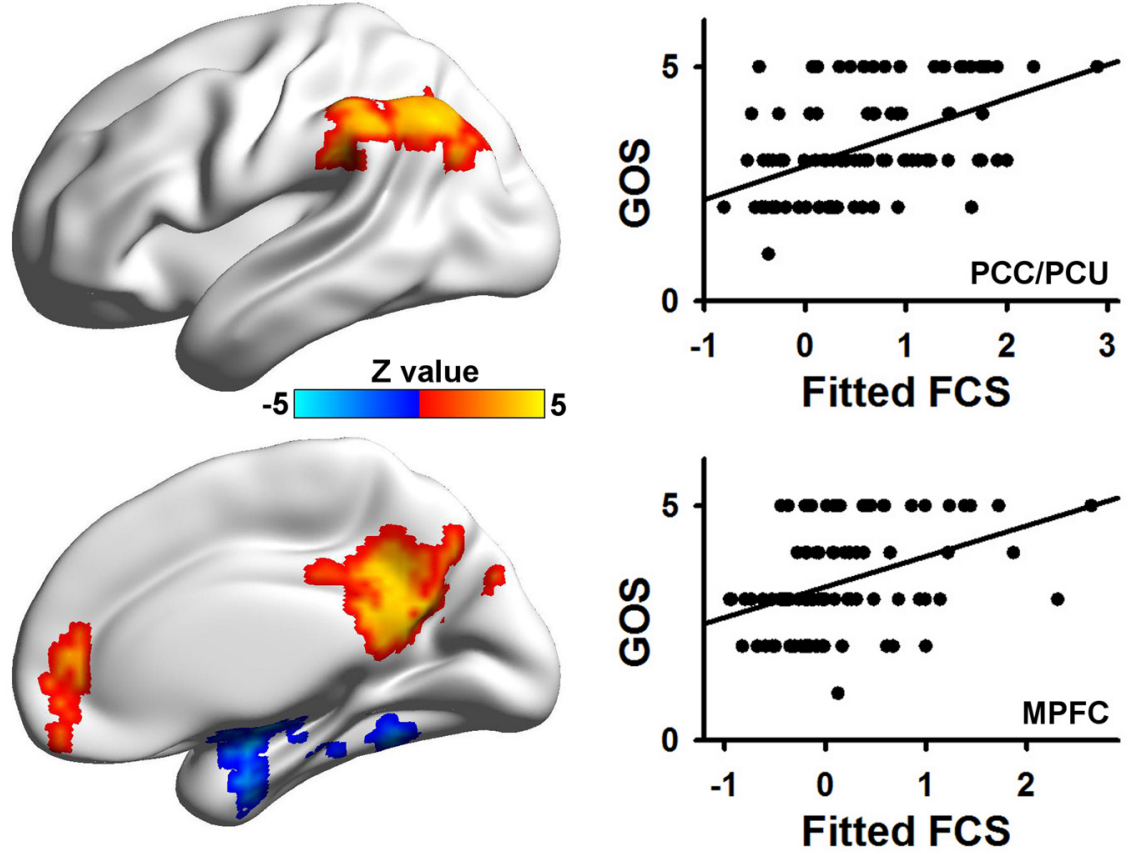

B FCS predicted dichotomized recovery outcome
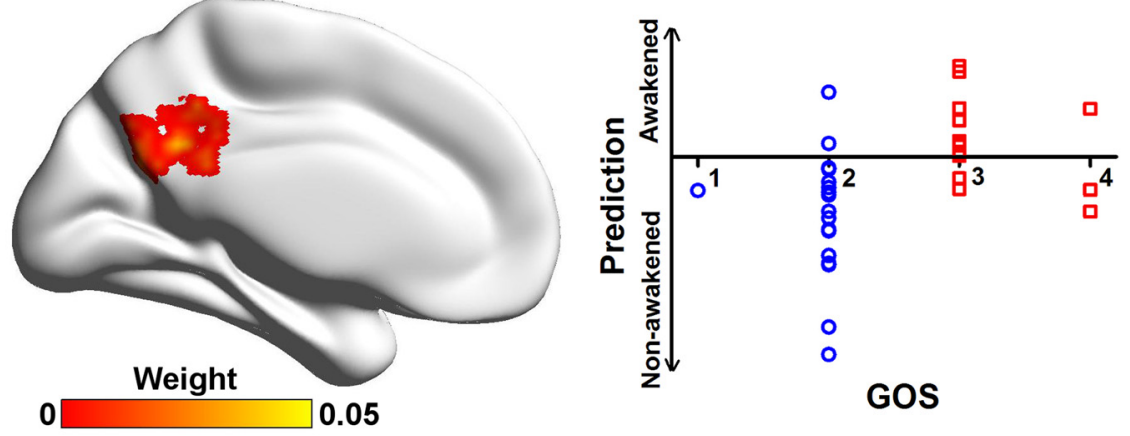

Figure 4. FCS predicted recovery outcome. $\boldsymbol{A}$, Five regions showed significant correlations with the GOS score $(p<0.05$ corrected). Scatter plots showing the difference between the mean fitted FCS in two representative regions (the PCC/PCU and the $\mathrm{MPFC}$ ) and the GOS score are shown. $\boldsymbol{B}$, Absolute discriminating maps of multivariate support vector machine analysis of UWS/NS plus coma patients to characterize awakened or nonawakened status 3 months after the $\mathrm{fMRI}$ scanning using FCS (clusters with at least 84 voxels were shown). The classifier with the LO0CV demonstrated accuracy of $81.25 \%$, a specificity of $88.89 \%$, and a sensitivity of $71.43 \%$. Scatter plot shows that 2 of 18 nonawakened patients (GOS score $<3$, labeled in blue circle) were incorrectly classified, and 4 of 14 awakened patients (GOS score $>2$, labeled in red square) were incorrectly classified. MPFC, Medial prefrontal cortex.

correlations and long-range connections. To exclude the potential influence of local autocorrelation on the FCS findings, we recomputed the FCS that set the very short-distance connections ( $<20 \mathrm{~mm}$ apart $)$ to zero (Power et al., 2011, 2013). At a given voxel, $x_{0}$, the FCS was recomputed as the average correlation between $x_{0}$ and other voxels that were $>20 \mathrm{~mm}$ from $x_{0}$. (6) The acquired brain injury patients experienced different types of etiology. Traumatic brain injury (TBI) patients comprised the predominant group, with 82 patients including 30 PC, 25 MCS, 17 UWS/ VS, and 10 coma patients (Table 1). We performed additional analyses on patients with TBI, including a main group effect from ANOVA and Spearman's correlation analyses. ANOVA was conducted among subjects in the control group with 34 healthy control subjects, and 4 TBI patient groups composed of 30 PC, 25 MCS, 17 UWS/VS, and 10 coma patients. Spearman's correlation analyses were performed on the data of 82 TBI patients between weighted FCS and clinical indices, including the GCS, CRS-R, and GOS.

\section{Results}

Demographic and clinical

characteristics

Table 2 represents the demographic and clinical features of all participants. There were no significant differences in gender $\left(\chi_{(4)}^{2}=3.84, p=0.43\right)$ or age $\left(F_{(4,128)}=0.45\right.$, $p=0.77)$ among the five groups. There were no significant differences in gender $\left(\chi_{(3)}^{2}=\right.$ $3.63, p=0.30)$, age $\left(F_{(3,95)}=0.42, p=\right.$ $0.74)$, or the time before $\mathrm{AMRI}$ after injury $\left(F_{(3,95)}=1.51, p=0.22\right)$ among the four acquired brain injury patient groups. Significant differences were observed among the four patient groups on the GCS $\left(F_{(3,95)}=146.32, p=1.73 \times 10^{-35}\right)$, the CRS-R $\left(F_{(3,95)}=252.97, p=3.71 \times\right.$ $\left.10^{-45}\right)$, and the GOS $\left(F_{(3,95)}=29.99, p=\right.$ $\left.9.94 \times 10^{-14}\right)$. Post hoc analyses showed significantly decreased GCS and CRS-R scores with reductions in consciousness in the PC, MCS, UWS/VS, and coma groups (all $p$ values $<0.05$ ), except between the UWS/VS and coma groups for the CRS-R $(p>0.05)$. The GOS scores of the MCS, UWS/VS, and coma groups were decreased compared with the PC patients, and in the UWS/VS group compared with the MCS group (all $p$ values $<0.05$ ). Of the 32 severe acquired brain injury patients (18 UWS/VS and 14 coma patients), 14 of them awoke within 3 months after the fMRI scanning (i.e., with GOS scores of at least 3, whereas the other 18 patients had GOS scores of $<3$; Table 2).

Disrupted functional connectivity strength in acquired brain injury The five groups showed higher FCS than the global mean, primarily in regions of the DMN (Greicius et al., 2003; Fox et al., 2005), including the PCC/PCU, the medial prefrontal cortex, and the inferior parietal lobule; in regions of the salience network ( $\mathrm{SN}$ )/executive control network (ECN; Fox et al., 2005; Seeley et al., 2007; Sridharan et al., 2008; Spreng, 2012), including the anterior insula, middle cingulate cortex, intraparietal sulcus, and middle and inferior frontal gyrus; and in the visual cortices (Fig. 1). Several regions, including the medial temporal lobe, inferior temporal gyrus, and basal ganglia, had lower FCS values than the global mean.

Significant differences in FCS among the five groups were primarily located in the DMN, including the PCC/PCU, medial prefrontal cortex, and inferior parietal lobule, and in the SN/ $\mathrm{ECN}$, including the middle cingulate cortex and anterior insula, and the intraparietal sulcus (Fig. 2A). Fingerprint plots (Fig. 2B) revealed that FCS decreased in the DMN and SN/ECN with loss of consciousness, and increased in the inferior temporal gyrus, medial temporal lobe, and basal ganglia. Post hoc analyses showed that these group effects were mainly driven by altered FCS in the four patient groups compared with the CONs, and in the MCS, 
Table 3. Demographic and clinical characteristics of the severe acquired brain injury patients awoke or not 3 months after fMRI acquisition

\begin{tabular}{lllc}
\hline & Awakened & Nonawakened & $p$ value \\
\hline Group & 6UWS/VS, 8 coma & 12 UWS/VS, 6 coma & 0.18 \\
Gender & $11 \mathrm{M}, 3 \mathrm{~F}$ & $12 \mathrm{M}, 6 \mathrm{~F}$ & 0.46 \\
Age, years & $35.4 \pm 11.6$ & $49.6 \pm 13.6$ & $\mathbf{0 . 0 0 4}$ \\
Time prior to fMRl after injury, d & $51.2 \pm 51.8$ & $75.8 \pm 61.7$ & 0.24 \\
GCS score & $7.1 \pm 1.1$ & $7.3 \pm 1.6$ & 0.61 \\
CRS-R score & $4.0 \pm 1.4$ & $4.2 \pm 1.9$ & 0.78 \\
GOS score & $3.2 \pm 0.4$ & $1.9 \pm 0.2$ & $<\mathbf{0 . 0 0 0 1}$ \\
\hline
\end{tabular}

Values are reported as the mean $\pm S D$, unless otherwise indicated. $p$ values $<0.05$ are labeled in bold.

UWS/VS, and coma groups compared with the PC group (Fig. $2 C-L)$. We found that $>90 \%$ of the regions that showed decreased FCS with loss of consciousness (i.e., the regions located at the DMN and SN/ECN) were functional hubs (i.e., the $20 \%$ of regions with the highest FCS from the control subjects), while $100 \%$ of those that showed increased FCS were non-hubs.

\section{Relationships among functional connectivity strength,} consciousness level, and recovery outcome in patients Using Spearman's correlation analysis, the resting-state FCS in the DMN, including the PCC/PCU, medial prefrontal cortex, and the left inferior parietal lobule, showed a significant positive correlation with the GCS score, while FCS in the medial temporal lobe/inferior temporal gyrus was negatively correlated with the GCS score (Fig. 3A). Similarly, FCS in the DMN, including the $\mathrm{PCC} / \mathrm{PCU}$ and left inferior parietal lobule, was positively correlated with CRS-R scores, whereas FCS in the medial temporal lobe/inferior temporal gyrus was negatively correlated with the CRS-R scores (Fig. 3B).

Resting-state FCS in the DMN, including the PCC/PCU, medial prefrontal cortex, and left inferior parietal lobule, and in the SN/ECN, including the intraparietal sulcus, positively correlated with recovery outcome, as assessed by the GOS. In addition, FCS in the medial temporal lobe/inferior temporal gyrus negatively correlated with the GOS (Fig. $4 A$ ). More than $80 \%$ of the regions that positively correlated with clinical indices were hubs, while $100 \%$ of regions that negatively correlated with clinical measures were non-hubs.

\section{Prediction of recovery outcome using functional connectivity strength}

Using the classifier trained on FCS and the LOOCV, support vector machine-based multivariate pattern analysis was able to predict whether or not UWS/VS plus coma patients regained consciousness in 3 months with an accuracy of $81.25 \%$, a sensitivity of $88.89 \%$, and a specificity of $71.43 \%$. The most discriminating features were located in the PCC/PCU (Fig. 4B). The PCC/ PCU region that could predict wakening completely overlapped with the hub regions. Neither clinical nor demographic variables, including the GCS, the CRS-R, gender, and time of fMRI after injury, were effective predictors, except for age (see Table 4). This underscores the value of resting-state functional connectivity strength as a tool for predicting future outcome.

As shown in Table 3, there was a significant age difference between patients who awoke within 3 months and those who did not; awakened patients were younger than nonawakened ones $(p=0.004)$. In addition, as shown in Table 4 , age could predict with high accuracy whether or not UWS/VS plus coma patients would be awakened. We controlled for age through both linear regression before multivariate support vector machine analysis
Table 4. Accuracy of multivariate support vector machine discrimination analysis on FCS and univariate receiver operating characteristic analysis on demographic and clinical variables

\begin{tabular}{llll}
\hline & \multicolumn{2}{l}{ Accuracy, $\%$} & \\
\cline { 2 - 4 } $\begin{array}{l}\text { Input for discrimination } \\
\text { analysis }\end{array}$ & $\begin{array}{l}\text { 18 Nonawakened } \\
\text { vs 14 awakened }\end{array}$ & $\begin{array}{l}\text { 13 Nonawakened } \\
\text { vs 14 awakened }\end{array}$ & $\begin{array}{l}\text { 9 Nonawakened } \\
\text { vs 14 awakened }\end{array}$ \\
\hline SVM (FCS) & & & $\mathbf{8 2 . 6 1}$ \\
LOOCV & $\mathbf{8 1 . 2 5}$ & $\mathbf{8 1 . 4 8}$ & $\mathbf{7 9 . 2 5}$ \\
LTOCV & $\mathbf{7 5 . 6 1}$ & $\mathbf{7 7 . 3 5}$ & \\
ROC & & & $\mathbf{7 8 . 2 6}$ \\
GCS & 50.00 & 51.85 & 69.57 \\
CRS-R & 59.38 & 62.96 & 69.57 \\
Time prior to fMRI & 68.75 & 70.37 & 65.22 \\
$\quad$ after injury (days) & & & 56.52 \\
Gender & 53.12 & 55.56 & $\mathbf{7 7 . 7 8}$ \\
Age, years & $\mathbf{8 1 . 2 5}$ &
\end{tabular}

ROC, Receiver operating characteristic; SVM, support vector machine. Accuracy $>75 \%$ is labeled in bold.

and by excluding the five oldest nonawakened patients; thus, there were no significant differences in age $(p=0.06)$. We repeated the discriminative analysis based on FCS and revealed a high prediction accuracy of $81.48 \%$ (Table 4 ) and key discrimination patterns at the PCC/PCU. In contrast with this sample, using age as an input decreased the rate of accurate prediction to $77.78 \%$ (Table 4). Furthermore, when we excluded the four oldest nonawakened patients (effect of age, $p=0.42$ ), there were minimal changes in classification accuracy (Table 4 , accuracy $=$ $82.61 \%$ ) and discriminative maps, using FCS as an input. However, with this smaller sample, age was not an effective predictor for dichotomized recovery outcome (Table 4).

Furthermore, a more detailed analysis using the LTOCV showed that the accuracy of wakening predictions was $>75 \%$ (Table 4). As expected, the accuracy of LTOCV was reduced compared with the LOOCV, which could be due to the smaller sample size used for feature selection.

\section{Specific PCC/PCU circuits correlating with consciousness level and predicting recovery outcome}

Convergent findings, including the main group effect of FCS, correlation with consciousness level and recovery, and discriminative power, were observed in the PCC/PCU. To identify specific circuits involved in the impairment of FCS in the $\mathrm{PCC} / \mathrm{PCU}$, we used it as a seed for resting-state functional connectivity analysis (Fig. 5A, inset). We showed that the PCC positively correlated with brain regions in the $\mathrm{DMN}$, and negatively correlated with regions in the SN/ECN (Fig. 5). Main group effects among the five groups were shown both in the DMN and SN/ECN (Fig. 6A). Fingerprint plots indicate that the strength of both positive and negative correlations with the PCC was decreased in the patients (Fig. 6B). Post hoc analyses showed that these group effects were mainly driven by altered functional connectivity in the four patient groups compared with the CONs, and in the MCS, UWS/VS, and coma groups compared with the PC group (Fig. $6 C-L$ ).

The positive correlation between the PCC/PCU and regions within the DMN significantly positively correlated with GCS, CRS-R (Figs. 7A, $8 A, B$ ), and GOS scores (Figs. $7 B, 8 C$ ), while the anticorrelation between the PCC and regions within the SN/ECN negatively correlated with clinical measurements (Figs. 7, 8).

As shown in Figures 6, 7, and 8, seed-based functional connectivity findings suggest that the disrupted FCS in the PCC/PCU was mainly driven by altered functional connectivity (1) within the DMN and (2) between the DMN and SN/ECN. 
A

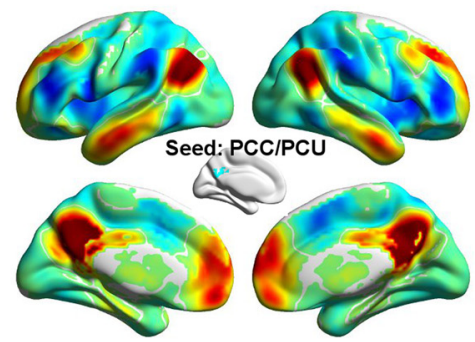

B $\quad$ PC
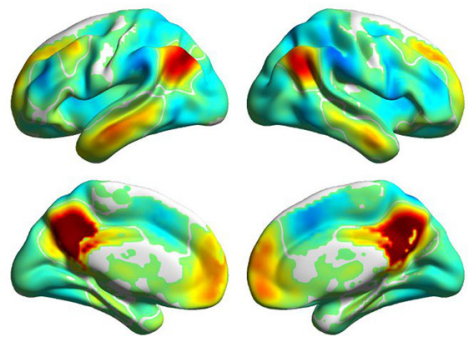

E

UWS/VS
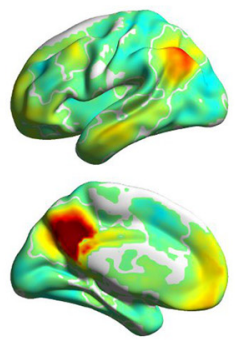
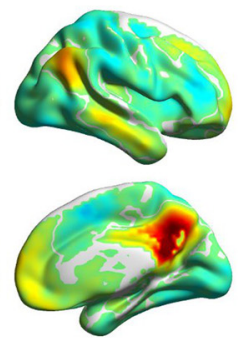
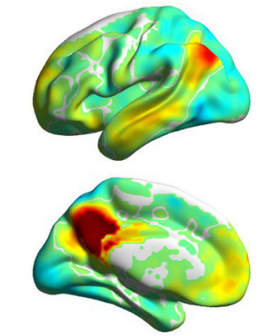

COMA

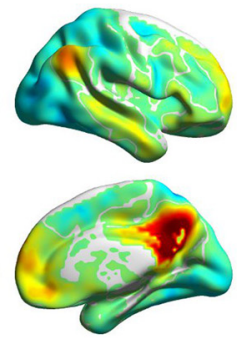

C MCS

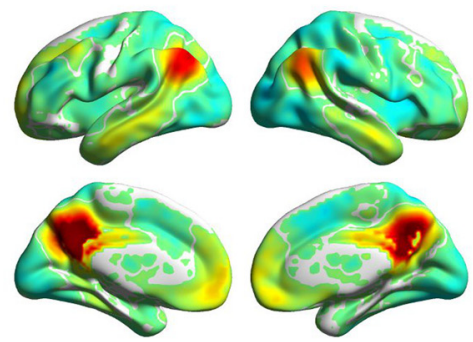

Figure 5. $\boldsymbol{A}-\boldsymbol{E}$, The $\mathrm{PCC} / \mathrm{PCU}$ resting-state functional connectivity (RSFC) maps of the CON $(\boldsymbol{A}), \mathrm{PC}(\boldsymbol{B}), \mathrm{MCS}(\boldsymbol{C})$, UWS/NS $(\boldsymbol{D})$, and coma $(\boldsymbol{E})$ groups.
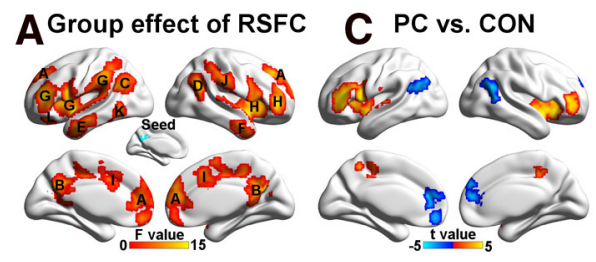

B Fingerprint plots of RSFC

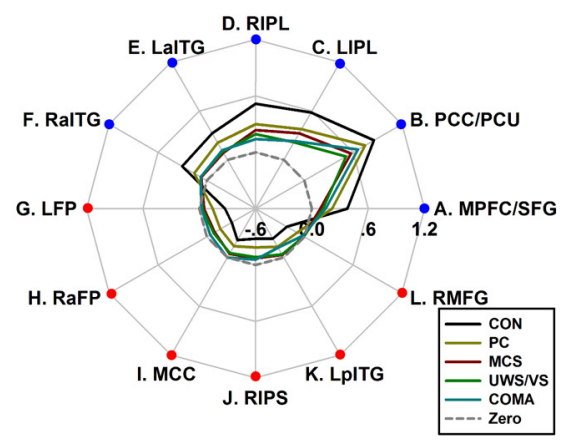

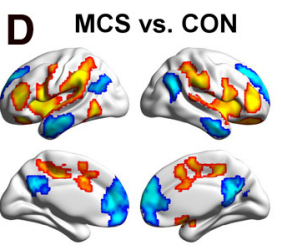

G MCs vs. PC
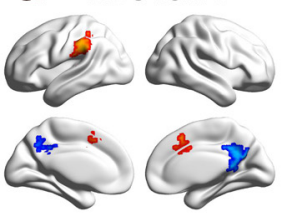

J UWS/vS vs. MCS
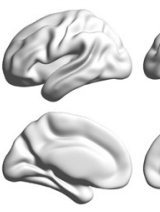

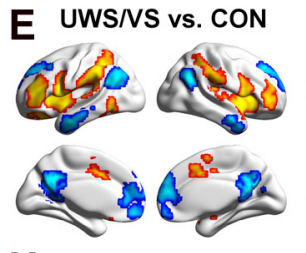

H uws/vs vs. PC
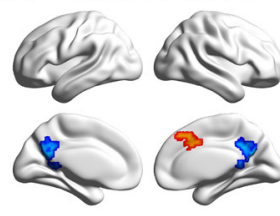

K COMA vs. Mcs
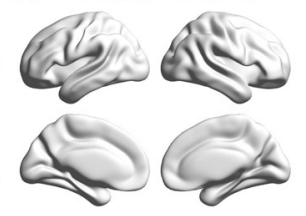
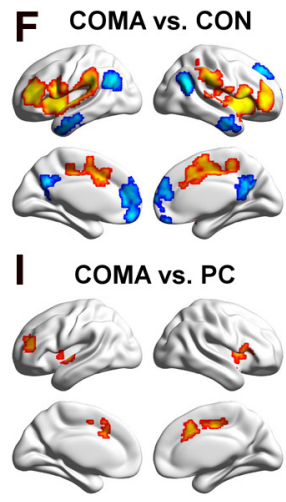

L COMA vs. UWS/vs
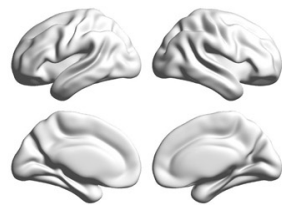

Figure 6. Group differences in the PCC/PCU resting-state functional connectivity (RSFC). $A$, Main group effect of the PCC/PCU RSFC among the five groups ( $p<0.05$ corrected). $\boldsymbol{B}$, Fingerprint plots of the mean fitted RSFCs of each region that showed a significant main effect across the five groups. Regions overlapped with the DMN are labeled in blue, while those overlapped with the SN/ECN are labeled in red. $(-L$, Post hoc comparisons of 10 pairs of groups $(p<0.05$ corrected); two-sample $t$-maps between $P C$ patients and CONs (C), MCS patients and CONs (D), UWS/VS patients and CONs $(\boldsymbol{E})$, coma patients and $C O N s(\boldsymbol{F}), M C S$ and $P C$ patients $(\boldsymbol{G})$, UWS/VS and PC patients $(\boldsymbol{H})$, coma and PC patients $(\boldsymbol{I})$, UWS/VS and MCS patients $(\boldsymbol{J})$, coma and MCS patients $(\boldsymbol{K})$, and between coma and UWS/VS patients $(\boldsymbol{L})$ were shown. Of note, there was no significant difference between UWS/VS and MCS patients $(\boldsymbol{J})$, between coma and MCS patients $(\boldsymbol{K})$, or between coma and UWS/VS patients (L). MPFC/SFG, Medial prefrontal cortex extending to the bilateral superior frontal gyrus; LIPL, left inferior parietal lobule; RIPL, right inferior parietal lobule; LalTG, left anterior inferior temporal gyrus; RalTG, right anterior inferior temporal gyrus; LFP, left frontoparietal regions, including the intraparietal sulcus, insula, middle and inferior frontal gyrus; RaFP, right anterior regions of FP, including the insula, middle and inferior frontal gyrus; MCC, middle cingulate cortex; RIPS, right intraparietal sulcus; LPITG, left posterior temporal gyrus; RMFG, right middle frontal gyrus.

Using the classifier trained on the PCC/PCU resting-state functional connectivity patterns, the support vector machine was able to correctly classify $64.29 \%$ of the patients who regained consciousness and $83.33 \%$ of the patients who did not, with an overall accuracy of $75 \%$. Discriminative regions were within the DMN, which positively correlated with the PCC, primarily concentrated in the PCC/PCU and the left anterior inferior temporal gyrus (Fig. 7B).
Validation results

As shown in Figure 9, the network type (Fig. 9B), data length (Fig. 9C), connection type (Fig. 9D), global mean signal removal (Fig. $9 E$ ), strong local connections (Fig. $9 F$ ), and type of acquired brain injury (Fig. $9 G$ ) showed minimal effects on group differences compared with our main findings (Fig. 9A). Different preprocessing and analysis strategies showed a negligible effect on Spearman's correlation with the GCS, the CRS-R, and the GOS 
scores, indicating that our results did not depend upon the analytic strategy used.

To show the presence and absence of significant differences among the analytic approaches, we overlapped the main group results from ANOVA with different approaches and determined the frequency of spatial overlap. A similar investigation of spatial overlap was conducted for the correlation analyses between FCS and clinical measurements. The most stable group effect of ANOVA was localized to the PCC/PCU, left intraparietal sulcus, basal ganglia, and medial temporal lobe, while correlations with the three clinical indices were robustly detected at the PCC/ PCU and the left inferior parietal lobule (Fig. 10).

\section{Discussion}

We performed whole-brain investigations of resting-state FCS in a large cohort of patients with loss of consciousness induced by acquired brain injury. We observed altered FCS in the DMN and SN/ECN of patients with a range of disease severities, including $\mathrm{PC}$, MCS, UWS/VS, and coma patients, compared with CONs. We found significant correlations between FCS in the DMN and level of consciousness. In addition, we found significant correlations between FCS in functional networks, including the $\mathrm{DMN}$ and $\mathrm{SN} / \mathrm{ECN}$, and recovery outcome. Importantly, FCS, mainly in the PCC/PCU, a major hub of the brain, discriminated whether patients experiencing UWS/VS and coma would be awakened within the following 3 months with an accuracy of $81.25 \%$. In addition, PCC/PCU resting-state functional connectivity to the DMN and SN/ECN predicted consciousness level and recovery outcome. Our findings suggest that the FCS of the PCC/ PCU and its functional connections to the DMN and SN/ECN may serve as potential diagnostic biomarkers for the clinical assessment of consciousness level and for the prediction of recovery outcome in acquired brain injury patients.

\footnotetext{
Disrupted resting-state functional connectivity strength with loss of consciousness

The healthy control subjects showed high FCS (i.e., functional hubs), mainly in regions of the DMN and SN/ECN, which was generally consistent with previous functional (Buckner et al., 2009; Liang et al., 2013; Tomasi et al., 2013) and structural (Hagmann et al., 2008; Gong et al., 2009) network studies. Using FCS as a reflection of global brain network connectivity, we investigated for the first time whether the impairment of connectivity associated with acquired brain injury was located at brain hubs or non-hubs, with a large sample of patients whose levels of consciousness varied. We showed decreased FCS associated with loss of consciousness mainly in these hub regions, indicating that acquired brain injury pathology specifically targeted functional hubs. The aberrant FCS of hub regions found in this study is supported by previous structural connectivity studies showing impaired white matter integrity of the DMN in patients with acquired brain injury (Sharp et
}

al., 2011; Fernández-Espejo et al., 2012). Our current largesample, seed-based, resting-state functional connectivity analyses, together with previous small-sample, resting-state fMRI studies, showed convergent decreased connectivity within the DMN (Boly et al., 2009; Vanhaudenhuyse et al., 2010; Norton et al., 2012), within the SN/ECN (Ham et al., 2014) and between the DMN and the SN/ECN (Boly et al., 2009) in patients with acquired brain injury. These functional connectivity analyses provided further evidence for the impairment of specific circuits that contributed to our FCS findings. Overall, the large patient populations with varied levels of consciousness included in the current study allowed us to draw much more definitive conclusions about the disruption of the DMN and SN/ECN in patients with acquired brain injury.

In contrast, increased FCS associated with loss of consciousness was shown in the medial temporal lobe, the inferior temporal gyrus, and the basal ganglia. Regions with increased FCS might present an adaptive or compensatory response to the loss of consciousness (Sharp et al., 2011; Achard et al., 2012; Caeyenberghs et al., 2012). Increased FCS in the medial temporal lobe associated with loss of consciousness is supported by a previous seed-based, resting-state functional connectivity study showing hyperlimbic connectivity between the medial temporal lobe and the ventral tegmental area in UWS/VS and MCS patients (Di Perri et al., 2013). Hyperconnectivity of the thalamus (part of the basal ganglia) has also been observed in patients with mild traumatic brain injury (Tang et al., 2011), which is consistent with our observation that increased FCS in the thalamus is associated with loss of consciousness. 

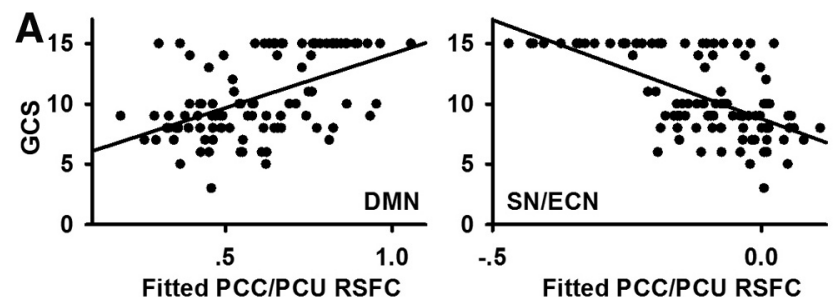

B
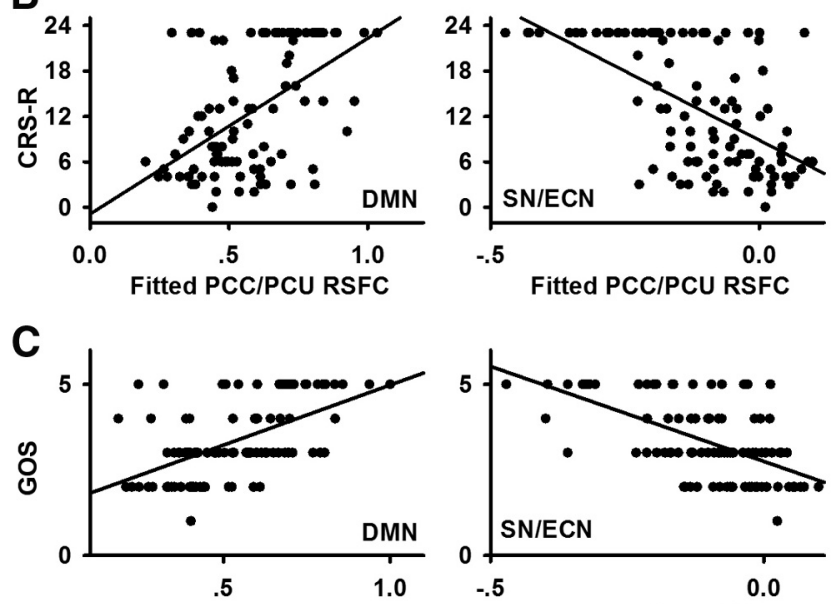

Fitted PCC/PCU RSFC

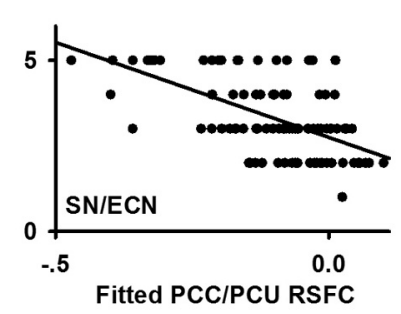

Figure 8. $\quad A-C$, Scatter plots demonstrate the relationship between $P C C / P C U$ resting-state functional connectivity (RSFC) and clinical indices, including GCS score ( $\boldsymbol{A})$, CRS-R score $(\boldsymbol{B})$, and GOS score (C). Figure 7 demonstrates that the regions that negatively correlated with clinical indices are located in the SN/ECN, while those that positively correlated with clinical indices are in the DMN. Thus, for each clinical index, all the voxels that positively correlated with that clinical index are defined as DMN, while those that negatively correlated with that clinical index are defined as SN/ECN. Scatter plots between clinical indices and mean fitted PCC/PCU RSFC (with covariates such as age, gender, and time of fMRl after injury removed) of the DMN are shown in the left column. Similarly, scatter plots between clinical indices and the mean fitted PCC/PCU RSFC of the SN/ECN are shown in the right column.

\section{Correlation between functional connectivity strength and} consciousness level

Previous studies have predominantly focused on differences in brain connectivity between groups under different consciousness states (Boly et al., 2009; Vanhaudenhuyse et al., 2010; Achard et al., 2012; Pandit et al., 2013). Relationships with consciousness levels indexed by the CRS-R or the GCS have seldom been reported, which might be because of the small sample sizes (Vanhaudenhuyse et al., 2010) and because certain important network features, such as FCS, have not been sufficiently emphasized in the previous studies. In this study, we showed that FCS of the DMN correlated with GCS and CRS-R scores, thus greatly expanding on previous small-sample studies (Vanhaudenhuyse et al., 2010). Thus, our findings suggest that disruptions of FCS in these specific regions might reflect the degree of consciousness loss, and contribute to our understanding of the neural mechanisms of consciousness and the pathophysiology of acquired brain injury

\section{Resting-state functional connectivity strength predicted recovery outcome in acquired brain injury}

Early and accurate prediction of the outcome for patients with loss of consciousness is frequently required for clinical care, prognosis, and medical-legal decision making (Lee et al., 2012; Sharp et al., 2014). Prediction of recovery outcome has been investigated using clinical and neuroimaging variables, which demon- strated that diffuse axonal injury was a potentially useful predictor (Lee et al., 2012). Recently, glucose metabolism of the frontoparietal cortices, assessed using ${ }^{18} \mathrm{~F}$-fluorodeoxyglucose PET, was shown to correctly predict recovery outcome with an accuracy of $74 \%$ (75 of 102 patients), whereas supplementary motor cortex and parahippocampal gyrus activation during a mental imagery task, assessed by fMRI, had a prediction accuracy of 53\% (36 of 65 patients; Stender et al., 2014). By examining resting-state functional connectivity, we showed that higher FCS in the DMN and SN/ECN during the baseline state predicted better recovery outcome in 3 months. Further, using multivariate support vector machine analysis, we showed that the PCC/PCU was a key region for predicting whether UWS/VS and coma patients would be awakened in 3 months, with $81.25 \%$ accuracy, and with both high sensitivity and specificity. The high accuracy (i.e., generalization rate of our discriminative analysis) would be informative for clinical care and medical-legal decision making. Those UWS/VS and coma patients predicted to be awakened in 3 months would be more likely to benefit from optimistic treatment decisions. This finding contrasted with the observations that age, gender, time before fMRI after brain injury, and the GCS and CRS-R scores cannot be used as effective predictors for GOS scores (Table 4).

The critical role of the PCC/PCU in consciousness recovery was supported by the finding that coma patients who subsequently regained consciousness showed preserved PCC/PCU resting-state activity, whereas it continued to be disrupted in those coma patients who did not recover (Norton et al., 2012). Furthermore, our analysis of seed-based resting-state functional connectivity of the PCC/PCU demonstrated that the strength of correlation within the DMN and anti-correlation of the DMN with the SN/ECN were crucial to the consciousness level and recovery outcome. The PCC/PCU is a central hub of the brain (Buckner et al., 2009; Liang et al., 2013; Leech and Sharp, 2014), with dense anatomical and functional connections (Hagmann et al., 2008; Buckner et al., 2009; Liang et al., 2013) and high baseline metabolism (Raichle et al., 2001; Liang et al., 2013). The PCC/ PCU has been shown to play a pivotal role in consciousness (Leech and Sharp, 2014). Here, we provide further evidence that this region is critical for consciousness recovery. Our findings imply that the intrinsic functional connectivity patterns of the posteromedial cortex might serve as general biomarkers and predictors of therapeutic recovery, especially in patients with acquired brain injury.

\section{Limitations and future work}

This study has several limitations. First, we included four groups of acquired brain injury patients, but the sample sizes for UWS/VS and coma were relatively small. Larger sample sizes for patients in all of the categories are needed for further studies; these studies would require multicenter collaborations. In addition, the radiological findings of lesion locations were heterogeneous across patients with acquired brain injury. The impact of the location and severity of a lesion on functional connectivity and consciousness loss needs further study using large samples. Second, although there was no significant difference in the time before fMRI after injury, there was a large difference in the average time since injury among different subgroups of patients, especially between coma and PC patients. However, we have controlled for the effect of time before fMRI through linear regression in the statistical model, and we will better match the time effect among subgroups in future studies by recruiting more PC patients with shorter times since injury. Finally, the PCC is highly 


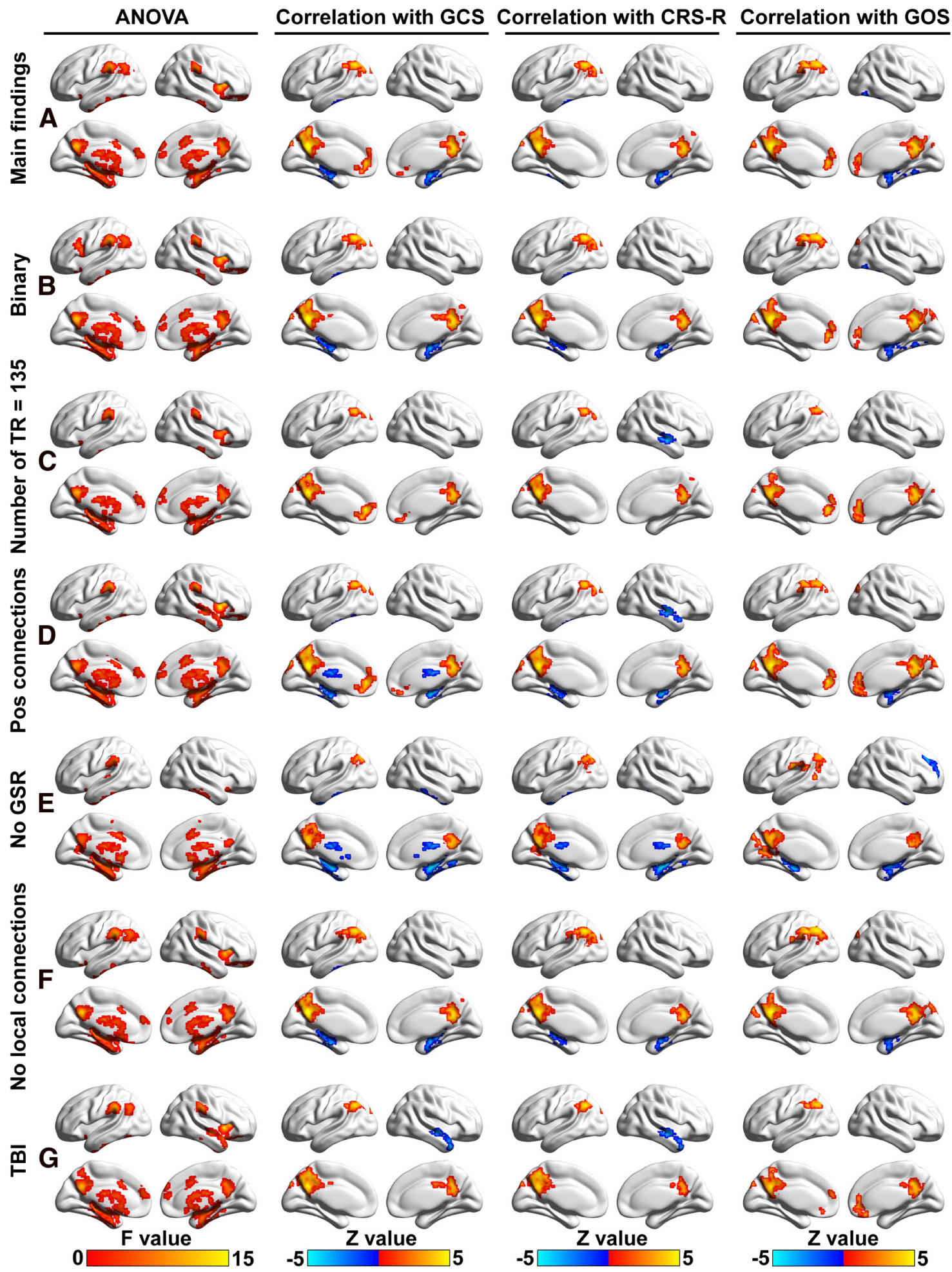

Figure 9. Validation of the FCS findings. $A-G$, Main group effect of FCS, and correlations between FCS and GCS, CRS-R, and GOS scores ( $p<0.05$ corrected) calculated by weighted network (our main findings; $\boldsymbol{A}$ ), binary network $(\boldsymbol{B})$, weighted network with shortest data length (number of TRs $=135 ; \boldsymbol{C}$, weighted network with only positive connections considered $(\boldsymbol{D})$, weighted network without global signal removal (GSR; $\boldsymbol{E}$ ), weighted network without local connections (excluded local connections that were within $20 \mathrm{~mm} ; \boldsymbol{F}$ ), and weighted network with TBI only (G).

heterogeneous (Leech et al., 2011; Leech and Sharp, 2014; Liang et al., 2015). Here we used a univariate seed-based approach to study the connectivity of the PCC, which might overlook its heterogeneous characteristics. In future studies, investigations of the functional connectivity of the PCC at a finer spatial scale and the use of multivariate approaches should be considered.

\section{Conclusions}

We included a large number of patients with varied levels of consciousness loss and matched control subjects in the current study, and demonstrated significant correlations between functional connectivity and clinical measurements. Furthermore, the current prediction analysis demonstrated the novel and strong 


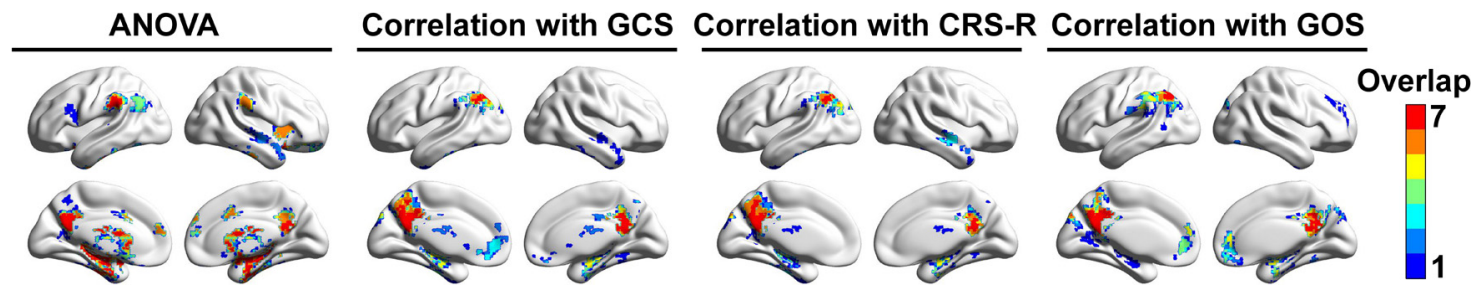

Figure 10. Frequencies of spatial overlap among the results of different preprocessing procedures in Figure 9.

prognostic value of the resting-state functional connectivity, which would be of potential clinical importance.

\section{References}

Achard S, Salvador R, Whitcher B, Suckling J, Bullmore E (2006) A resilient, low-frequency, small-world human brain functional network with highly connected association cortical hubs. J Neurosci 26:63-72. CrossRef Medline

Achard S, Delon-Martin C, Vértes PE, Renard F, Schenck M, Schneider F, Heinrich C, Kremer S, Bullmore ET (2012) Hubs of brain functional networks are radically reorganized in comatose patients. Proc Natl Acad Sci U S A 109:20608-20613. CrossRef Medline

Belcher AM, Yen CC, Stepp H, Gu H, Lu H, Yang Y, Silva AC, Stein EA (2013) Large-scale brain networks in the awake, truly resting marmoset monkey. J Neurosci 33:16796-16804. CrossRef Medline

Boly M, Tshibanda L, Vanhaudenhuyse A, Noirhomme Q, Schnakers C, Ledoux D, Boveroux P, Garweg C, Lambermont B, Phillips C, Luxen A, Moonen G, Bassetti C, Maquet P, Laureys S (2009) Functional connectivity in the default network during resting state is preserved in a vegetative but not in a brain dead patient. Hum Brain Mapp 30:2393-2400. CrossRef Medline

Buckner RL, Sepulcre J, Talukdar T, Krienen FM, Liu H, Hedden T, AndrewsHanna JR, Sperling RA, Johnson KA (2009) Cortical hubs revealed by intrinsic functional connectivity: mapping, assessment of stability, and relation to Alzheimer's disease. J Neurosci 29:1860-1873. CrossRef Medline

Bullmore E, Sporns O (2009) Complex brain networks: graph theoretical analysis of structural and functional systems. Nat Rev Neurosci 10:186198. CrossRef Medline

Caeyenberghs K, Leemans A, Heitger MH, Leunissen I, Dhollander T, Sunaert S, Dupont P, Swinnen SP (2012) Graph analysis of functional brain networks for cognitive control of action in traumatic brain injury. Brain 135:1293-1307. CrossRef Medline

Cole MW, Pathak S, Schneider W (2010) Identifying the brain's most globally connected regions. Neuroimage 49:3132-3148. CrossRef Medline

Cox RW (1996) AFNI: software for analysis and visualization of functional magnetic resonance neuroimages. Comput Biomed Res 29:162-173. CrossRef Medline

Dai Z, Yan C, Wang Z, Wang J, Xia M, Li K, He Y (2012) Discriminative analysis of early Alzheimer's disease using multi-modal imaging and multi-level characterization with multi-classifier (M3). Neuroimage 59: 2187-2195. CrossRef Medline

Di Perri C, Bastianello S, Bartsch AJ, Pistarini C, Maggioni G, Magrassi L, Imberti R, Pichiecchio A, Vitali P, Laureys S, Di Salle F (2013) Limbic hyperconnectivity in the vegetative state. Neurology 81:1417-1424. CrossRef Medline

Dosenbach NU, Nardos B, Cohen AL, Fair DA, Power JD, Church JA, Nelson SM, Wig GS, Vogel AC, Lessov-Schlaggar CN, Barnes KA, Dubis JW, Feczko E, Coalson RS, Pruett JR Jr, Barch DM, Petersen SE, Schlaggar BL (2010) Prediction of individual brain maturity using fMRI. Science 329: 1358-1361. CrossRef Medline

Ecker C, Rocha-Rego V, Johnston P, Mourao-Miranda J, Marquand A, Daly EM, Brammer MJ, Murphy C, Murphy DG, Consortium MA (2010) Investigating the predictive value of whole-brain structural MR scans in autism: a pattern classification approach. Neuroimage 49:44-56. CrossRef Medline

Fernández-Espejo D, Soddu A, Cruse D, Palacios EM, Junque C, Vanhaudenhuyse A, Rivas E, Newcombe V, Menon DK, Pickard JD, Laureys S, Owen AM (2012) A role for the default mode network in the bases of disorders of consciousness. Ann Neurol 72:335-343. CrossRef Medline
Forman SD, Cohen JD, Fitzgerald M, Eddy WF, Mintun MA, Noll DC (1995) Improved assessment of significant activation in functional magnetic resonance imaging (fMRI): use of a cluster-size threshold. Magn Reson Med 33:636-647. CrossRef Medline

Fox MD, Snyder AZ, Vincent JL, Corbetta M, Van Essen DC, Raichle ME (2005) The human brain is intrinsically organized into dynamic, anticorrelated functional networks. Proc Natl Acad Sci U S A 102:9673-9678. CrossRef Medline

Fox MD, Zhang D, Snyder AZ, Raichle ME (2009) The global signal and observed anticorrelated resting state brain networks. J Neurophysiol 101: 3270-3283. CrossRef Medline

Friston KJ, Williams S, Howard R, Frackowiak RS, Turner R (1996) Movement-related effects in fMRI time-series. Magn Reson Med 35:346355. CrossRef Medline

Giacino JT, Ashwal S, Childs N, Cranford R, Jennett B, Katz DI, Kelly JP, Rosenberg JH, Whyte J, Zafonte RD, Zasler ND (2002) The minimally conscious state: definition and diagnostic criteria. Neurology 58:349353. CrossRef Medline

Giacino JT, Kalmar K, Whyte J (2004) The JFK Coma Recovery Scale-Revised: measurement characteristics and diagnostic utility. Arch Phys Med Rehabil 85:2020-2029. CrossRef Medline

Gong G, He Y, Concha L, Lebel C, Gross DW, Evans AC, Beaulieu C (2009) Mapping anatomical connectivity patterns of human cerebral cortex using in vivo diffusion tensor imaging tractography. Cereb Cortex 19:524536. CrossRef Medline

Greicius MD, Krasnow B, Reiss AL, Menon V (2003) Functional connectivity in the resting brain: a network analysis of the default mode hypothesis. Proc Natl Acad Sci U S A 100:253-258. CrossRef Medline

Hagmann P, Cammoun L, Gigandet X, Meuli R, Honey CJ, Wedeen VJ, Sporns O (2008) Mapping the structural core of human cerebral cortex. PLoS Biol 6:e159. CrossRef Medline

Ham TE, Bonnelle V, Hellyer P, Jilka S, Robertson IH, Leech R, Sharp DJ (2014) The neural basis of impaired self-awareness after traumatic brain injury. Brain 137:586-597. CrossRef Medline

He Y, Evans A (2010) Graph theoretical modeling of brain connectivity. Curr Opin Neurol 23:341-350. CrossRef Medline

He Y, Dagher A, Chen Z, Charil A, Zijdenbos A, Worsley K, Evans A (2009) Impaired small-world efficiency in structural cortical networks in multiple sclerosis associated with white matter lesion load. Brain 132:33663379. CrossRef Medline

Jennett B, Bond M (1975) Assessment of outcome after severe brain damage. Lancet 1:480-484. Medline

Långsjö JW, Alkire MT, Kaskinoro K, Hayama H, Maksimow A, Kaisti KK, Aalto S, Aantaa R, Jääskeläinen SK, Revonsuo A, Scheinin H (2012) Returning from oblivion: imaging the neural core of consciousness. J Neurosci 32:4935-4943. CrossRef Medline

Laureys S, Owen AM, Schiff ND (2004) Brain function in coma, vegetative state, and related disorders. Lancet Neurol 3:537-546. CrossRef Medline

Lee SY, Kim SS, Kim CH, Park SW, Park JH, Yeo M (2012) Prediction of outcome after traumatic brain injury using clinical and neuroimaging variables. J Clin Neurol 8:224-229. CrossRef Medline

Leech R, Sharp DJ (2014) The role of the posterior cingulate cortex in cognition and disease. Brain 137:12-32. CrossRef Medline

Leech R, Kamourieh S, Beckmann CF, Sharp DJ (2011) Fractionating the default mode network: distinct contributions of the ventral and dorsal posterior cingulate cortex to cognitive control. J Neurosci 31:3217-3224. CrossRef Medline

Liang X, Zou Q, He Y, Yang Y (2013) Coupling of functional connectivity and regional cerebral blood flow reveals a physiological basis for network 
hubs of the human brain. Proc Natl Acad Sci U S A 110:1929-1934. CrossRef Medline

Liang X, Zou Q, He Y, Yang Y (2015) Topologically reorganized connectivity architecture of default-mode, executive-control, and salience networks across working memory task loads. Cereb Cortex. Advance online publication. Retrieved 17 August 2015. doi:10.1093/cercor/bhu316. CrossRef Medline

Murphy K, Birn RM, Handwerker DA, Jones TB, Bandettini PA (2009) The impact of global signal regression on resting state correlations: are anticorrelated networks introduced? Neuroimage 44:893-905. CrossRef Medline

Norton L, Hutchison RM, Young GB, Lee DH, Sharpe MD, Mirsattari SM (2012) Disruptions of functional connectivity in the default mode network of comatose patients. Neurology 78:175-181. CrossRef Medline

Pandit AS, Expert P, Lambiotte R, Bonnelle V, Leech R, Turkheimer FE, Sharp DJ (2013) Traumatic brain injury impairs small-world topology. Neurology 80:1826-1833. CrossRef Medline

Plum F, Posner JB (1966) The diagnosis of stupor and coma. New York: F.A. Davis.

Power JD, Cohen AL, Nelson SM, Wig GS, Barnes KA, Church JA, Vogel AC, Laumann TO, Miezin FM, Schlaggar BL, Petersen SE (2011) Functional network organization of the human brain. Neuron 72:665-678. CrossRef Medline

Power JD, Barnes KA, Snyder AZ, Schlaggar BL, Petersen SE (2012) Spurious but systematic correlations in functional connectivity MRI networks arise from subject motion. Neuroimage 59:2142-2154. CrossRef Medline

Power JD, Schlaggar BL, Lessov-Schlaggar CN, Petersen SE (2013) Evidence for hubs in human functional brain networks. Neuron 79:798-813. CrossRef Medline

Power JD, Mitra A, Laumann TO, Snyder AZ, Schlaggar BL, Petersen SE (2014) Methods to detect, characterize, and remove motion artifact in resting state fMRI. Neuroimage 84:320-341. CrossRef Medline

Raichle ME, MacLeod AM, Snyder AZ, Powers WJ, Gusnard DA, Shulman GL (2001) A default mode of brain function. Proc Natl Acad Sci U S A 98:676-682. CrossRef Medline

Schiff ND, Nauvel T, Victor JD (2014) Large-scale brain dynamics in disorders of consciousness. Curr Opin Neurobiol 25:7-14. CrossRef Medline

Schnakers C (2012) Clinical assessment of patients with disorders of consciousness. Arch Ital Biol 150:36-43. CrossRef Medline

Seeley WW, Menon V, Schatzberg AF, Keller J, Glover GH, Kenna H, Reiss AL, Greicius MD (2007) Dissociable intrinsic connectivity networks for salience processing and executive control. J Neurosci 27:2349-2356. CrossRef Medline

Sharp DJ, Beckmann CF, Greenwood R, Kinnunen KM, Bonnelle V, De Boissezon X, Powell JH, Counsell SJ, Patel MC, Leech R (2011) Default mode network functional and structural connectivity after traumatic brain injury. Brain 134:2233-2247. CrossRef Medline

Sharp DJ, Scott G, Leech R (2014) Network dysfunction after traumatic brain injury. Nat Rev Neurol 10:156-166. CrossRef Medline
Spreng RN (2012) The fallacy of a "task-negative" network. Front Psychol 3:145. CrossRef Medline

Sridharan D, Levitin DJ, Menon V (2008) A critical role for the right frontoinsular cortex in switching between central-executive and default-mode networks. Proc Natl Acad Sci U S A 105:12569-12574. CrossRef Medline

Stender J, Gosseries O, Bruno MA, Charland-Verville V, Vanhaudenhuyse A, Demertzi A, Chatelle C, Thonnard M, Thibaut A, Heine L, Soddu A, Boly M, Schnakers C, Gjedde A, Laureys S (2014) Diagnostic precision of PET imaging and functional MRI in disorders of consciousness: a clinical validation study. Lancet 384:514-522. CrossRef Medline

Tang L, Ge Y, Sodickson DK, Miles L, Zhou Y, Reaume J, Grossman RI (2011) Thalamic resting-state functional networks: disruption in patients with mild traumatic brain injury. Radiology 260:831-840. CrossRef Medline

Teasdale G, Jennett B (1974) Assessment of coma and impaired consciousness. A practical scale. Lancet 2:81-84. Medline

The Multi-Society Task Force on PVS (1994) Medical aspects of the persistent vegetative state (1). N Engl J Med 330:1572-1579. CrossRef Medline

Tomasi D, Wang GJ, Volkow ND (2013) Energetic cost of brain functional connectivity. Proc Natl Acad Sci U S A 110:13642-13647. CrossRef Medline

Tononi G (2004) An information integration theory of consciousness. BMC Neurosci 5:42. CrossRef Medline

Tononi G, Koch C (2008) The neural correlates of consciousness: an update. Ann N Y Acad Sci 1124:239-261. CrossRef Medline

van den Heuvel MP, Sporns O (2013) Network hubs in the human brain. Trends Cogn Sci 17:683-696. CrossRef Medline

Vanhaudenhuyse A, Noirhomme Q, Tshibanda LJ, Bruno MA, Boveroux P, Schnakers C, Soddu A, Perlbarg V, Ledoux D, Brichant JF, Moonen G, Maquet P, Greicius MD, Laureys S, Boly M (2010) Default network connectivity reflects the level of consciousness in non-communicative braindamaged patients. Brain 133:161-171. CrossRef Medline

Wang JH, Zuo XN, Gohel S, Milham MP, Biswal BB, He Y (2011) Graph theoretical analysis of functional brain networks: test-retest evaluation on short- and long-term resting-state functional MRI data. PLoS One 6:e21976. CrossRef Medline

Wang L, Saalmann YB, Pinsk MA, Arcaro MJ, Kastner S (2012) Electrophysiological low-frequency coherence and cross-frequency coupling contribute to BOLD connectivity. Neuron 76:1010-1020. CrossRef Medline

Xia M, Wang J, He Y (2013) BrainNet Viewer: a network visualization tool for human brain connectomics. PLoS One 8:e68910. CrossRef Medline

Zeman A (2001) Consciousness. Brain 124:1263-1289. CrossRef Medline

Zou Q, Ross TJ, Gu H, Geng X, Zuo XN, Hong LE, Gao JH, Stein EA, Zang YF, Yang Y (2013) Intrinsic resting-state activity predicts working memory brain activation and behavioral performance. Hum Brain Mapp 34:32043215. CrossRef Medline

Zuo XN, Ehmke R, Mennes M, Imperati D, Castellanos FX, Sporns O, Milham MP (2012) Network centrality in the human functional connectome. Cereb Cortex 22:1862-1875. CrossRef Medline 